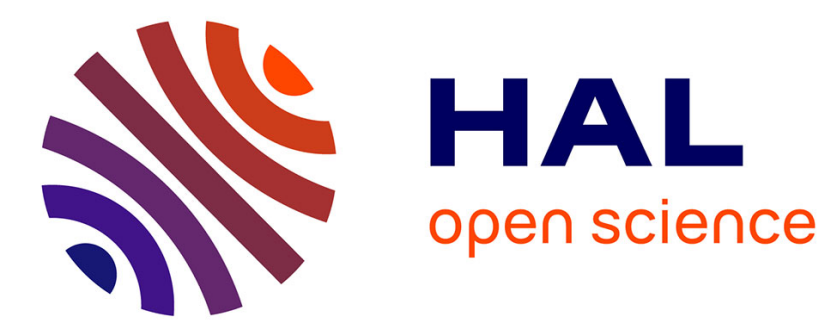

\title{
Phénomènes de relaxation dans les structures planes épitaxiales à l'arséniure de gallium
}

P. Rossel, Henri Tranduc, Jacques Graffeuil, C. Azizi

\section{To cite this version:}

P. Rossel, Henri Tranduc, Jacques Graffeuil, C. Azizi. Phénomènes de relaxation dans les structures planes épitaxiales à l'arséniure de gallium. Revue de Physique Appliquée, 1977, 12 (10), pp.1679-1694. 10.1051/rphysap:0197700120100167900 . jpa-00244387

\section{HAL Id: jpa-00244387 https://hal.science/jpa-00244387}

Submitted on 1 Jan 1977

HAL is a multi-disciplinary open access archive for the deposit and dissemination of scientific research documents, whether they are published or not. The documents may come from teaching and research institutions in France or abroad, or from public or private research centers.
L'archive ouverte pluridisciplinaire HAL, est destinée au dépôt et à la diffusion de documents scientifiques de niveau recherche, publiés ou non, émanant des établissements d'enseignement et de recherche français ou étrangers, des laboratoires publics ou privés. 
Classification

Physics Abstracts

$72.20-72.80 \mathrm{E}-73.40$

$73.60 \mathrm{~F}-73.90-77.40$

\title{
PHÉNOMẼNES DE RELAXATION DANS LES STRUCTURES PLANES ÉPITAXIALES A L'ARSÉNIURE DE GALLIUM
}

\author{
P. ROSSEL, H. TRANDUC, J. GRAFFEUIL et C. AZIZI \\ Laboratoire d'Automatique et d'Analyse des Systèmes \\ du Centre National de la Recherche Scientifique \\ 7, avenue Colonel-Roche, 31400 Toulouse, France
}

(Reçu le 18 avril 1977, accepté le 28 juin 1977)

\begin{abstract}
Résumé. - Les phénomènes de relaxation se produisant en basses fréquences dans les structures planes épitaxiales, avec ou sans grille, en arséniure de gallium sur substrat semi-isolant sont analysés. Il est montré expérimentalement qu'il existe diverses caractéristiques courant-tension qui sont fonction de l'amplitude, de la dépendance temporelle de la tension appliquée et de la température d'essai. Par ailleurs, en petits signaux, l'admittance de sortie des dispositifs est assimilable à un réseau électrique du premier ordre. Une analyse, basée sur les propriétés de la double charge d'espace à l'interface semiconducteur semi-isolant associées à l'équation de transport dans le semiconducteur permet d'expliquer l'existence des caractéristiques limite courant tension et la forme circulaire du diagramme d'admittance. Une application à la détermination des résistances thermiques de ces structures est proposée.

Abstract. - The low frequency relation phenomena that take place on both gated and ungated gallium arsenide planar epitaxial structure are discussed. The voltage-current characteristics are shown to be highly dependent upon: the amplitude, the time dependence of the applied voltage and the temperature. For small signals the output impedance of the device can be described by a first order electric network.

Both, the circular form of the admittance diagram and the display of a variety of voltage current shapes are analyzed in terms of the interface double space charge properties associated to the semiconductor transfert equation. An application to the thermal resistance determination, for these structures; is suggested.
\end{abstract}

1. Introduction. - Dans les transistors hyperfréquences à effet de champ à grille métallique et à l'arseniure de gallium, la région active est une couche semiconductrice épitaxiée sur un substrat semi-isolant. Les améliorations technologiques apportées au processus d'épitaxie ont agi sur les qualités de l'interface semiconducteur - semi-isolant et ont permis d'éviter la dégradation de la mobilité des porteurs au voisinage de cet interface que l'on observait sur les premières structures [1]. Les dispositifs actuels sont encore le siège de phénomènes mal expliqués. Ceuxci affectent les performances aussi bien dans le domaine des basses fréquences que dans celui des hyperfréquences. Leur liste a été dressée par Barrera [2]. En basses fréquences, ils se traduisent par l'existence des boucles sur les caractéristiques statiques, par de faibles valeurs de la transconductance et par les effets de la polarisation de substrat. En hautes fréquences, le problème le plus important est lié à la faible valeur de la résistance de sortie.

Afin de mieux comprendre l'origine de certains de ces phénomènes, nous proposons dans cet article une étude des mécanismes de relaxation se produisant en basses fréquences sur les dispositifs à structure plane en arséniure de gallium. Les propriétés que l'on observe expérimentalement, à savoir d'une part l'existence de diverses caractéristiques courant-tension et d'autre part la dégradation de l'admittance de sortie lorsque la fréquence augmente, seront tout d'abord décrites. Il sera ensuite montré théoriquement compte tenu du mécanisme d'ionisation des centres profonds dans le substrat qu'il existe une infinité de caractéristiques de courant qui se situent entre deux courbes limites correspondant à deux états de charge particuliers dans ce substrat. Au moyen d'une analyse dynamique de la cinétique des charges d'espace, les propriétés fonctionnelles de l'admittance de sortie seront expliquées et un schéma électrique équivalent de cette admittance sera proposé. Enfin, les conséquences et applications de ces mécanismes de relaxation seront discutées.

2. Propriétés expérimentales. - Les structures étudiées sont d'une part des éléments résistifs constitués de couches d'arseniure de gallium $\mathrm{N}$ d'épaisseur comprise entre 0,3 et $1 \mu \mathrm{m}$ de dopage variant entre $10^{16}$ et $10^{17}$ atomes $/ \mathrm{cm}^{3}$ et réalisés par épitaxie en phase vapeur sur des substrats semi-isolants d'arsé- 


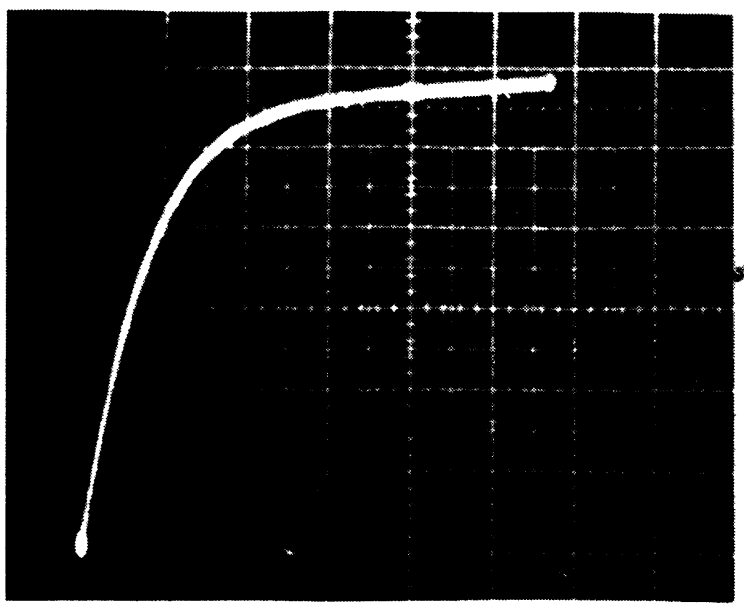

1a) $\mathrm{F}=0,1 \mathrm{~Hz}$

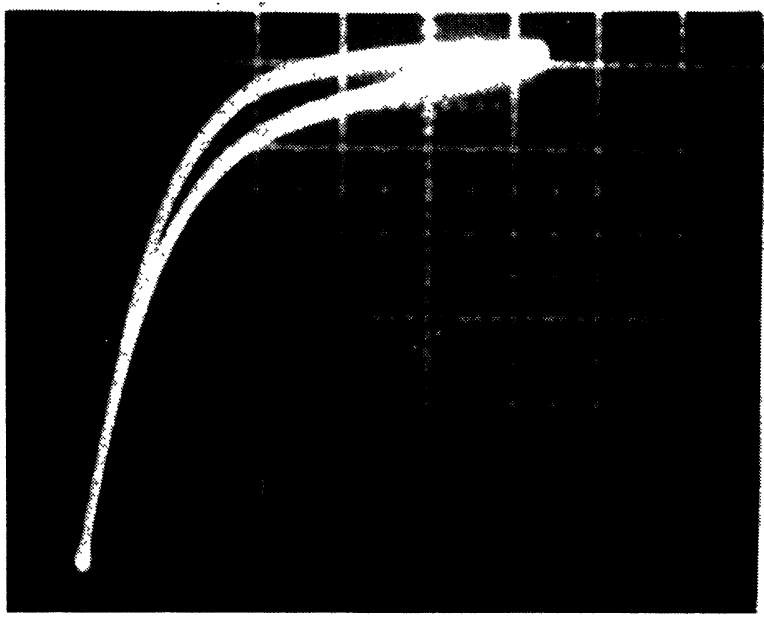

1c) $\mathrm{F}=100 \mathrm{~Hz}$

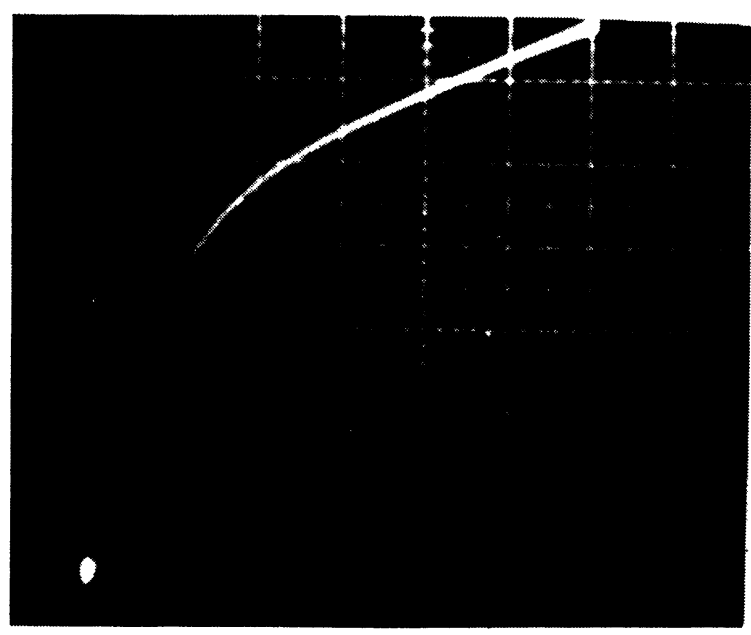

l(c) F: 10 hH,

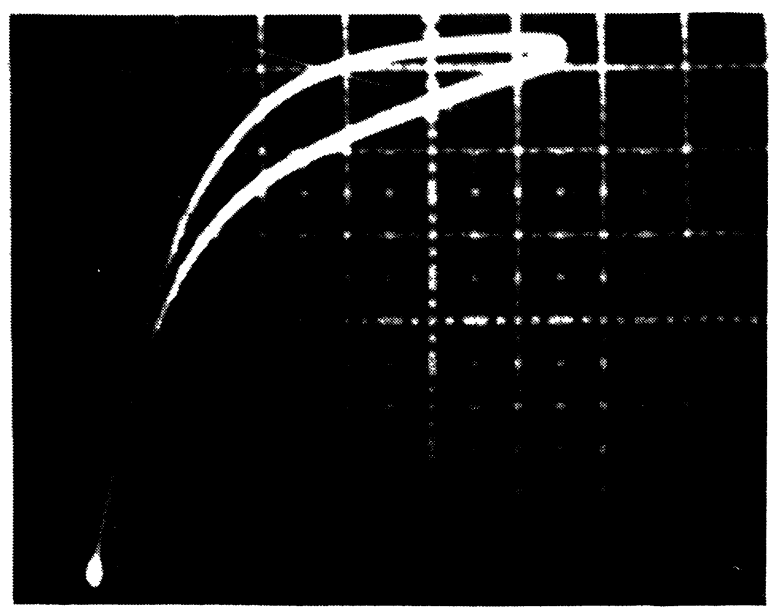

1b) $\mathrm{F} \stackrel{\circ}{=} 10 \mathrm{~Hz}$

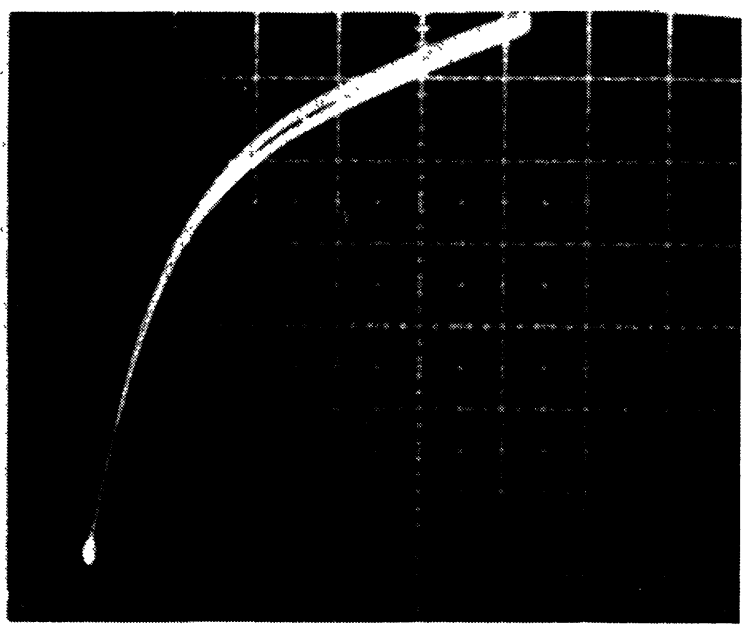

1d) $\mathrm{F}=1 \mathrm{kHz}$

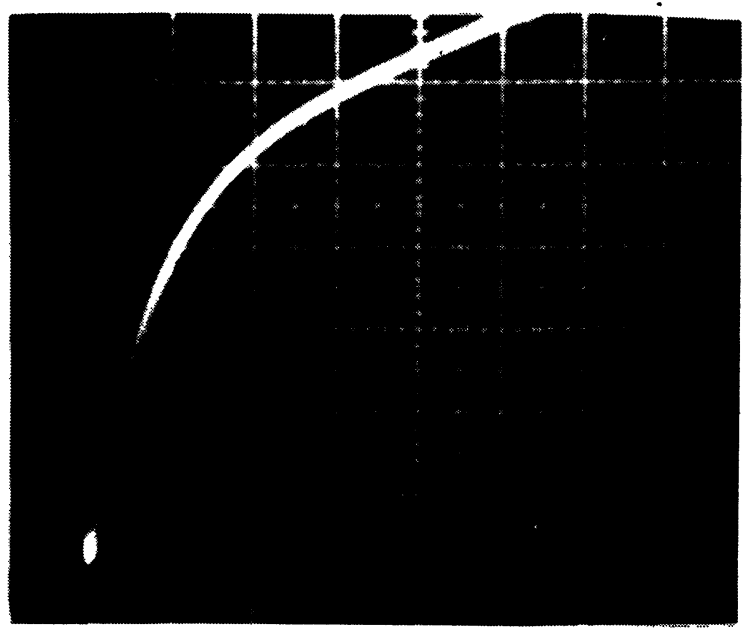

1/) $1 \quad 100 \mathrm{hH} /$

Fig. 1. - Caractéristiques $I_{\mathrm{D}}\left(V_{\mathrm{D}}\right)$ d'une structure avec grille, GAT 1, 396A 21. Tension de drain demi-sinusoïdale, $T_{\mathrm{a}}=300 \mathrm{~K}$. Horizontal : $V_{\mathrm{D}}, 1$ volt/div. Vertical $: I_{\mathrm{D}}, 2 \mathrm{~mA} /$ div. $V_{\mathrm{G}}=0$.

[Current Voltage characteristics $I_{\mathrm{D}}\left(V_{\mathrm{D}}\right)$ obtained with a half sine wave drain voltage; MESFET GAT $1 ; T_{\mathrm{a}}=300 \mathrm{~K}$. Horizontal $: 1 \mathrm{~V} / \mathrm{Div}$. Vertical : $2 \mathrm{~mA} /$ Div. $V_{\mathrm{G}}=0$.] 
niure de gallium dopés au chrome. Les contacts ohmiques obtenus par alliage or-germanium sont distants de $L=6 \mu \mathrm{m}$. Le deuxième type de structure est constitué par des transistors à effet de champ, à grille d'aluminium sur couche d'arséniure de gallium $\mathbf{N}$ dopée entre $5 \times 10^{16}$ et $2 \times 10^{17}$ atomes $/ \mathrm{cm}^{3}$ et sur substrats dopés au chrome. Les longueurs $L$ des grilles varient entre 1 et $4 \mu \mathrm{m}$. Les largeurs $Z$ des structures sont comprises entre 100 et $500 \mu \mathrm{m}$.

2.1. COMPortement EN LARges Signaux. - Lorsque sur ces deux types de structures la tension de drain varie selon une loi demi-sinusoïdale du temps, il apparaît que la caractéristique courant draintension dépend de la valeur de la fréquence. En régime statique ou de très basses fréquences (Fig. 1a), la caractéristique sature pour les valeurs élevées de la tension de drain. Lorsque la fréquence augmente des boucles d'hystéresis apparaissent (Fig. 1b à 1d), puis disparaissent en hautes fréquences (Fig. 1e et 1f). Dans ce dernier cas, le courant de drain est supérieur à celui relevé en régime continu. La gamme de fréquences pour laquelle il existe une boucle d'hystéresis dépend de la structure, mais surtout de la température d'essai. A la température ambiante, cette gamme est située entre quelques hertz et quelques centaines de kilohertz.

A la température de l'azote liquide, le cycle d'hystéresis apparaît, mais avec des constantes de temps

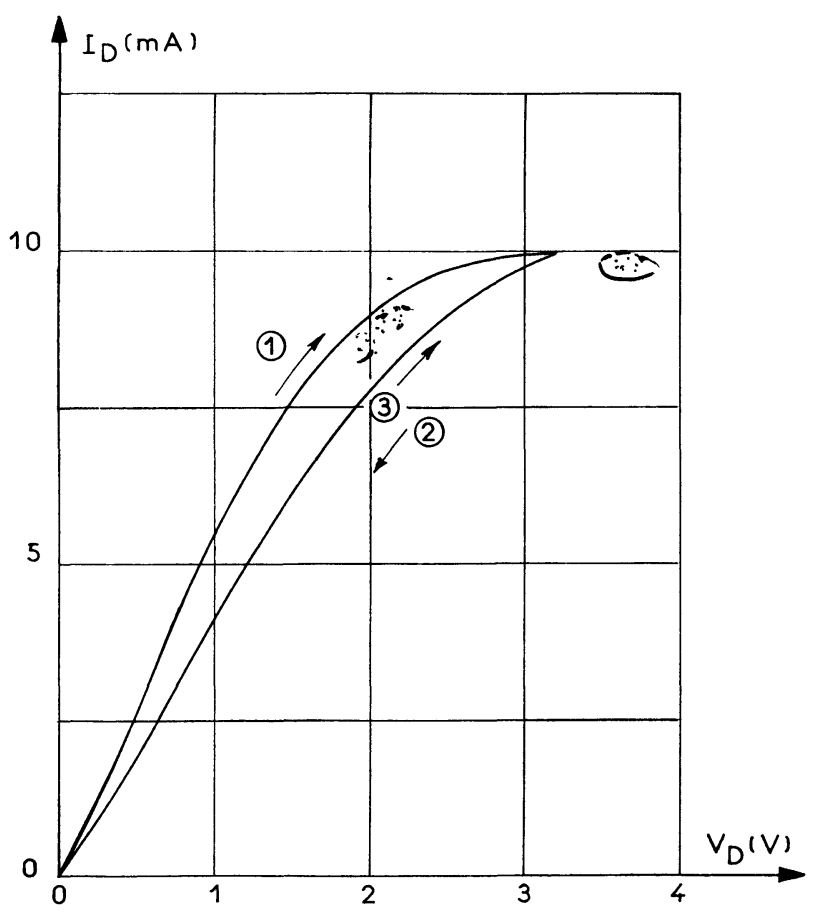

FIG. 2.1. - Caractéristique $I_{\mathrm{D}}\left(V_{\mathrm{D}}\right)$. Structure sans grille $\mathrm{TH} \mathrm{n}^{\circ} 2$ $T_{\mathrm{a}}=-196^{\circ} \mathrm{C}$ 1) montée en tension 2) descente en tension 3) deuxième montée.

[Current Voltage characteristics $I_{\mathrm{D}}\left(V_{\mathrm{D}}\right)$. Ungated device TH no. 2 ; $T_{\mathrm{a}}=-196^{\circ} \mathrm{C}$; 1) First increase of voltage ; 2) First decrease of voltage ; 3) Second increase of the voltage.] très longues. A titre d'exemple, la figure 2.1. représente un cycle relevé sur une structure sans grille lorsque la tension de drain est une fonction rampe, avec des temps de montée et de descente égaux à 600 secondes. La courbe 1 correspond à la première montée de tension drain, la courbe 2 au retour à la tension nulle. Une fois ce cycle $(1 \rightarrow 2)$ effectué, il est nécessaire de replacer l'échantillon à la température ambiante en polarisation nulle, puis de le refroidir, pour pouvoir obtenir à nouveau la caractéristique 1. Si cette condition n'est pas respectée, la remontée suit la courbe 3 confondue avec 2 . Enfin, on notera qu'il existe une infinité de cycles d'hystéresis caractérisés par les retours $2,2^{\prime} \ldots$, qui dépendent de la valeur de tension de drain maximale atteinte lors du tracé 1 (Fig. 2.2.).

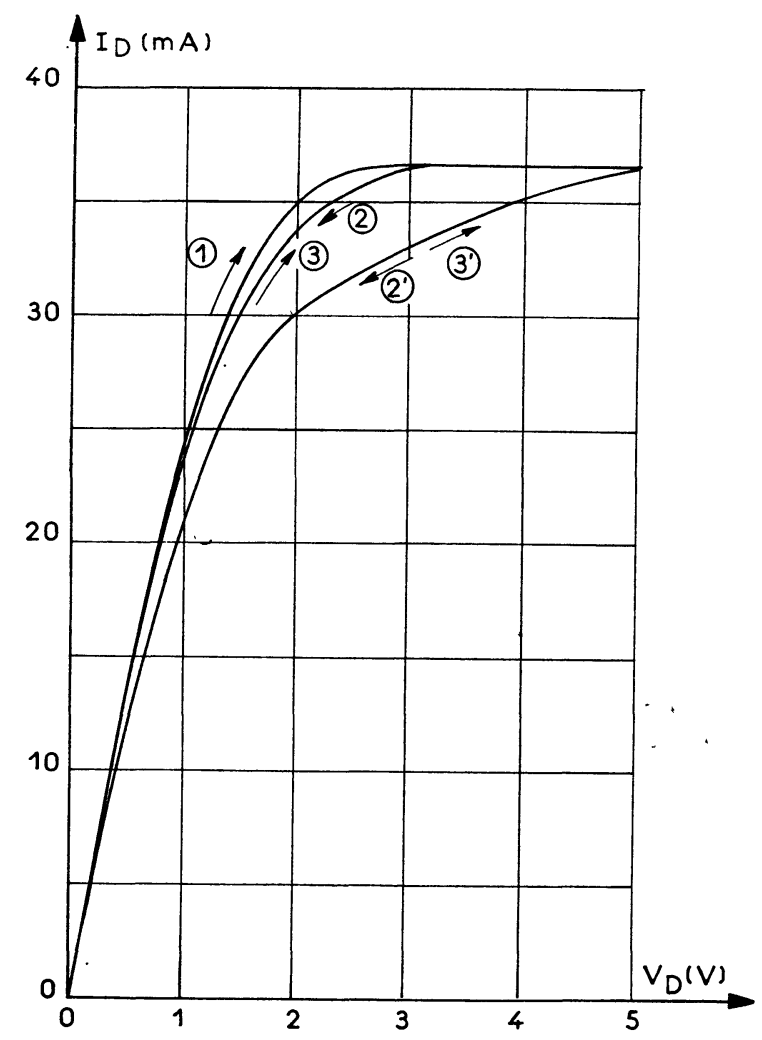

FIG. 2.2. - Caractéristique $I_{\mathrm{D}}\left(V_{\mathrm{D}}\right)$. Structure avec grille, GAT 2, 347 B. $T_{\mathrm{a}}=-196^{\circ} \mathrm{C} ; V_{\mathrm{G}}=0 \mathrm{~V}$.

[Current-Voltage characteristics $I_{\mathrm{D}}\left(V_{\mathrm{D}}\right)$. MESFET GAT 2. $T_{\mathrm{a}}=-196^{\circ} \mathrm{C} ; V_{\mathrm{G}}=0 \mathrm{~V}$.]

2.2. Comportement EN PETITS SIGNAUX:- - En vue d'analyser les propriétés de cette dépendance fréquentielle, la conductance de sortie en petits signaux des structures est mesurée au moyen d'un pont de Nernst (Fig. 3). La structure est placée dans l'un des bras et est polarisée en continu par les sources $E_{1}$ et $E_{2}$. Dans le bras adjacent, la valeur de la résistance et de la capacité, à l'équilibre du pont, fournissent les parties réelle $\operatorname{Re}\left(Y_{22}\right)$ et imagi- 


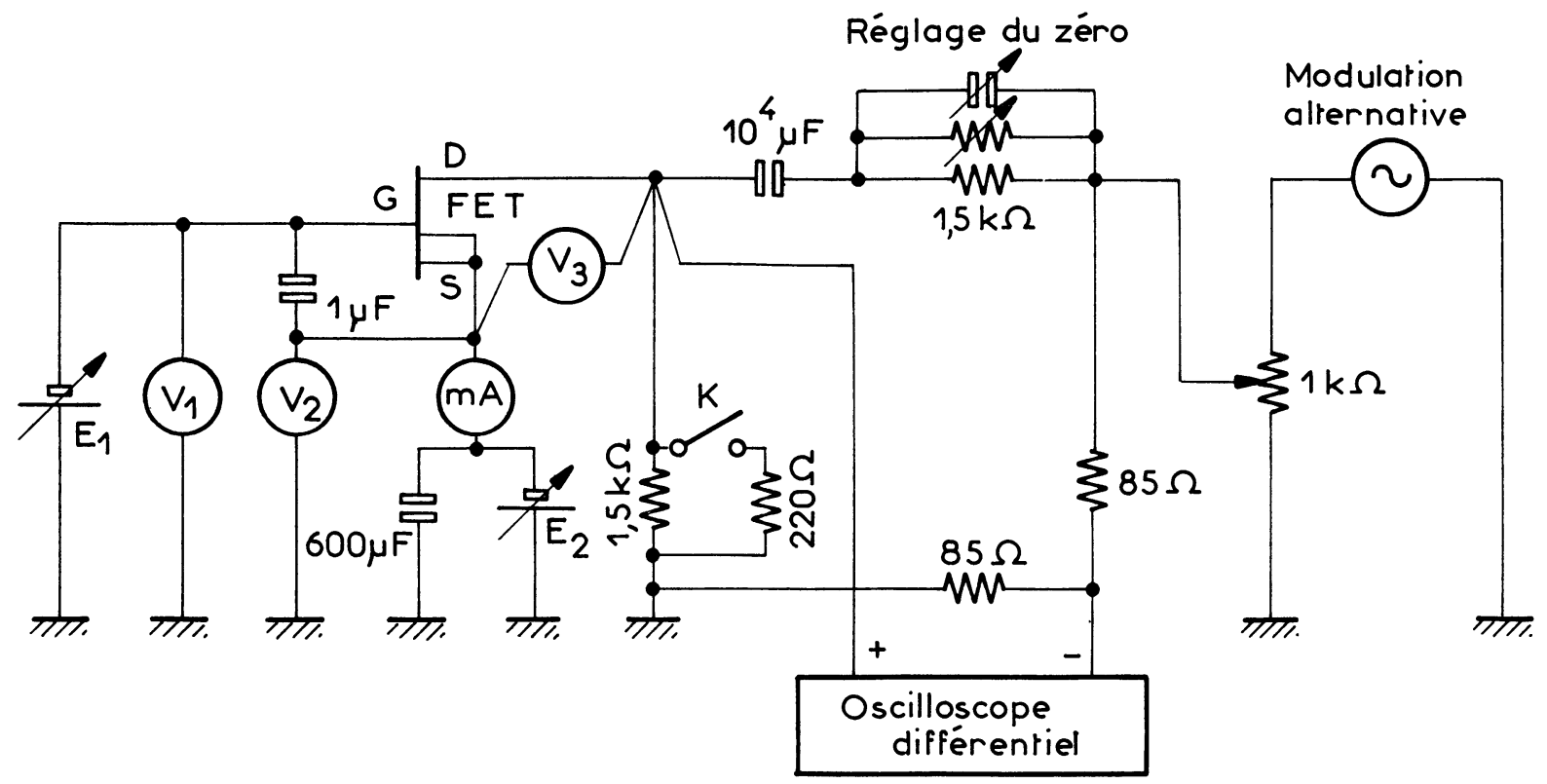

FIG. 3. - Schéma de mesure de l'admittance de sortie des structures.

[Schematic measuring set of the output admittance.]

naire $\operatorname{Im}\left(Y_{22}\right)$ de cette admittance. Les mesures sont effectuées entre $1 \mathrm{~Hz}$ et $500 \mathrm{kHz}$.

Pour toutes les structures testées, avec ou sans grille, il a été observé que, à conditions de polarisation et de température fixées :

- L'impédance de sortie est fonction décroissante de la fréquence.

- Dans le plan de Nyquist, les variations de la partie imaginaire de l'admittance de sortie en fonction de la partie réelle, la fréquence étant le paramètre, sont un demi-cercle (Figs. 4-5). Le diamètre et la position du centre dépendent des tensions continues de polarisation. Ce cercle existe quels que soient la nature et le régime de fonctionnement du dispositif, soit en régime de saturation pour les fortes valeurs de la tension drain source, soit en régime ohmique.

- L'identification de cette admittance montre que celle-ci est équivalente à un réseau du premier ordre (Fig. 6). En effet, le tracé du rapport $\operatorname{Im}\left(Y_{22}\right) / \omega$ en fonction de $\operatorname{Re}\left(Y_{22}\right)$ est une droite. Un exemple de détermination expérimentale des éléments constitutifs de ce réseau est illustré par la figure 6. La constante de temps $\mathfrak{6}^{*}$ associée à ce réseau correspond à la fréquence pour laquelle la partie imaginaire de l'admittance $Y_{22}$ est maximale.

- Cette constante de temps est une fonction exponentielle croissante de l'inverse de la température absolue du cristal d'arséniure de gallium. Son énergie d'activation est égale à $0,7 \mathrm{eV}$ (Fig. 7).

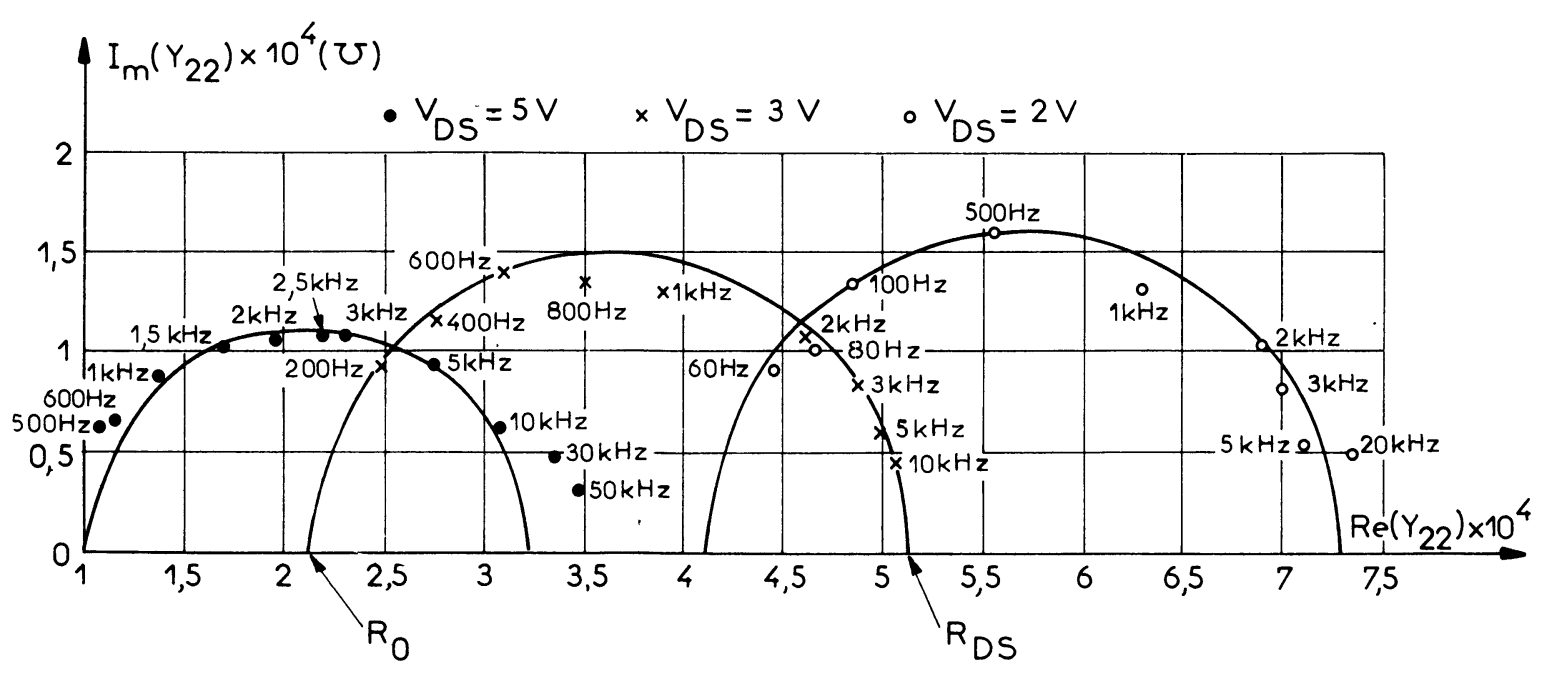

FIG. 4.1. - Evolutions en basses fréquences de l'admittance de sortie. Structure TH 7 GF 6 avec grille $\left(V_{\mathrm{Gs}}=0\right) ; T_{\mathrm{a}}=300 \mathrm{~K}$. [Low frequency variations of the output admittance. MESFET TH 7 GF $6, V_{\mathrm{G}}=0 \mathrm{~V} ; T_{\mathrm{a}}=300 \mathrm{~K}$.] 


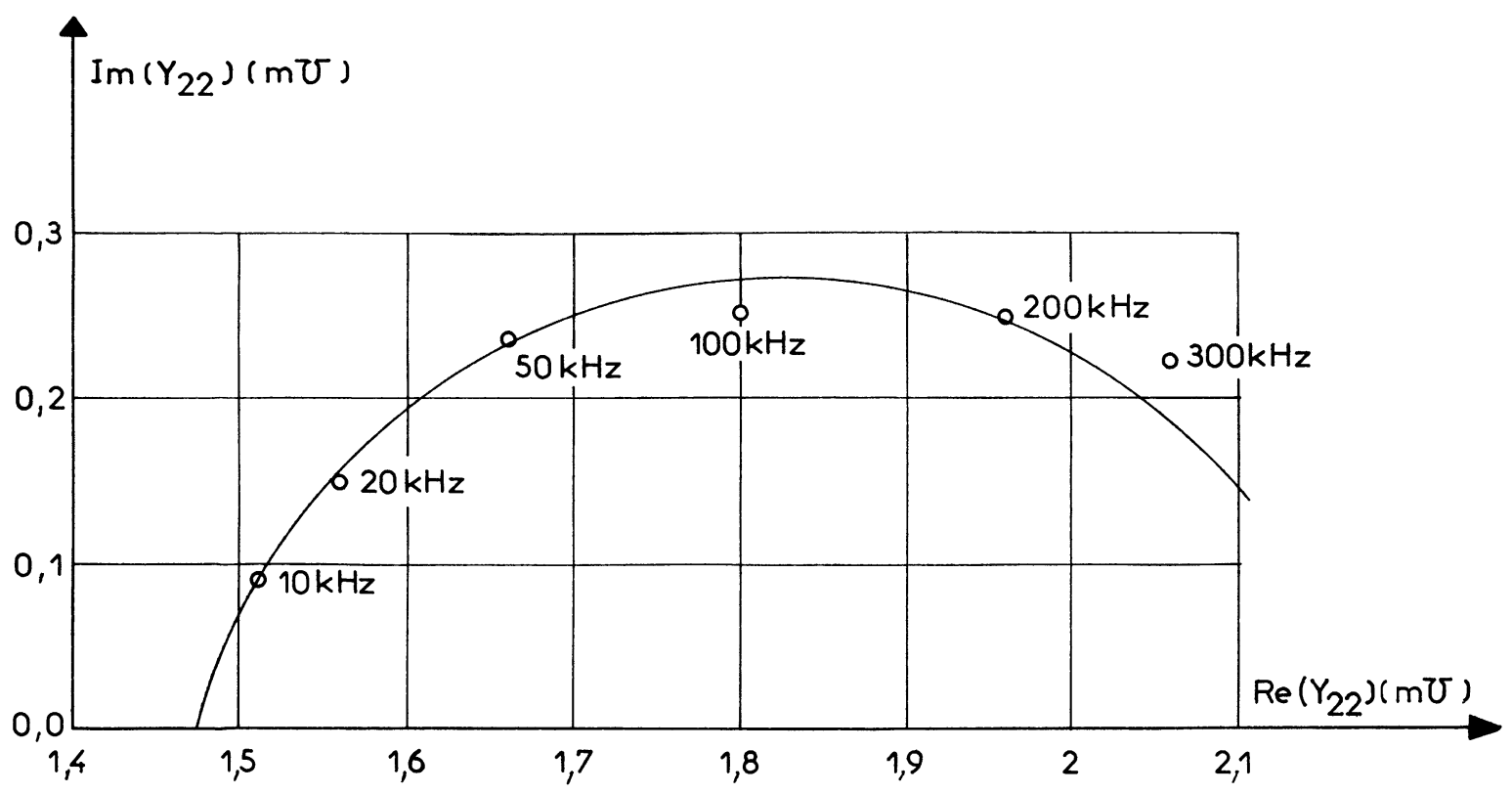

Fig. 4.2. - Variation de l'admittance de sortie en basses fréquences. Structure NEC V 244, $V_{\mathrm{D}}=3 \mathrm{~V} ; V_{\mathrm{G}}=-0,8 \mathrm{~V} ; I_{\mathrm{D}}=24 \mathrm{~mA}$; $T_{\mathrm{a}}=300 \mathrm{~K}$.

[Low frequency variation of the output admittance. MESFET NEC V 244, $V_{\mathrm{D}}=3 \mathrm{~V} ; V_{\mathrm{G}}=-0.8 \mathrm{~V} ; I_{\mathrm{D}}=24 \mathrm{~mA} ; T_{\mathrm{a}}=300 \mathrm{~K}$.]

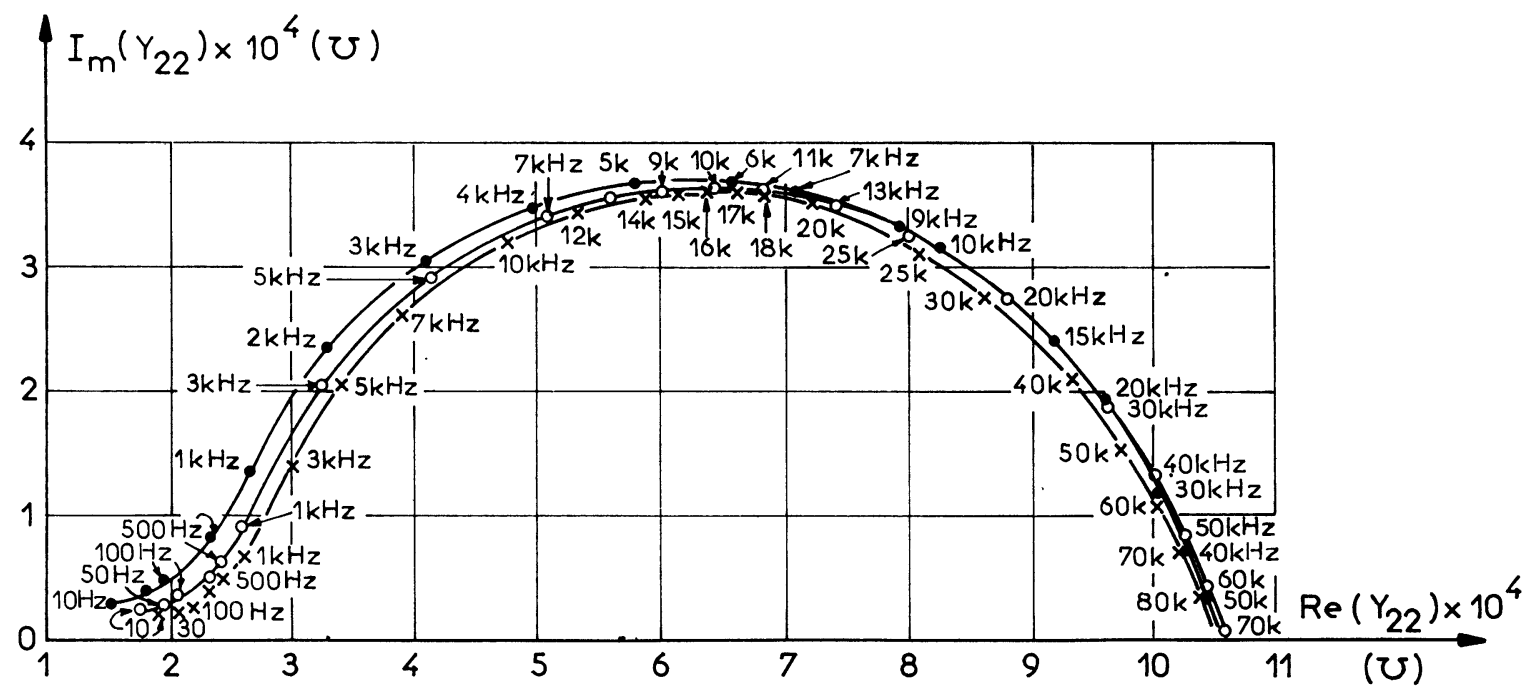

FIG. 4.3. - Variations de l'admittance de sortie en fonction de la fréquence et de la température. Structure avec grille GAT $2413 ; V_{\mathbf{D}}=5 \mathrm{~V}$; $V_{\mathrm{GS}}=0 ; \bullet T_{\mathrm{a}}=300 \mathrm{~K} ; \circ T_{\mathrm{a}}=310 \mathrm{~K} ; \times T_{\mathrm{a}}=320 \mathrm{~K}$.

[Low frequency variations of the output admittance at various temperatures. MESFET GAT $2 ; V_{\mathrm{D}}=5 \mathrm{~V} ; V_{\mathrm{G}}=0 \mathrm{~V} ; \bullet T_{\mathrm{a}}=300 \mathrm{~K}$; $O T_{\mathrm{a}}=310 \mathrm{~K} ; \times T_{\mathrm{a}}=320 \mathrm{~K}$.]

3. Interprétation des phénomènes. - 3.1. GÉNÉRALITÉs. - Le comportement thermique exponentiel des constantes de temps associées au diagramme circulaire d'admittance et l'existence de diverses caractéristiques courant-tension stables à la température de l'azote liquide ne peuvent être expliqués par toute hypothèse de cycles thermiques dûs à des mécanismes d'échauffement du cristal.

L'origine des phénomènes étudiés doit être recherchée dans un mécanisme de piégeage qui se produit dans une région particulière de la structure. Si l'on. se réfère aux principaux résultats publiés visant à décrire l'effet des pièges sur les caractéristiques électriques des transistors à effet de champ sur Gas As, on trouve tout d'abord un premier mécanisme proposé par Adlestein [8] qui est lié à la cinétique de centres pièges dans la zone dépeuplée, sous la grille métallique. Comme le mécanisme de relaxation se produit sur des couches sans grille, ne comportant pas cette zone dépeuplée, cette hypothèse est à exclure. Asai et al. [3] utilisant un modèle électrostatique classique du transistor ont tẹté d'expliquer la dégradation 


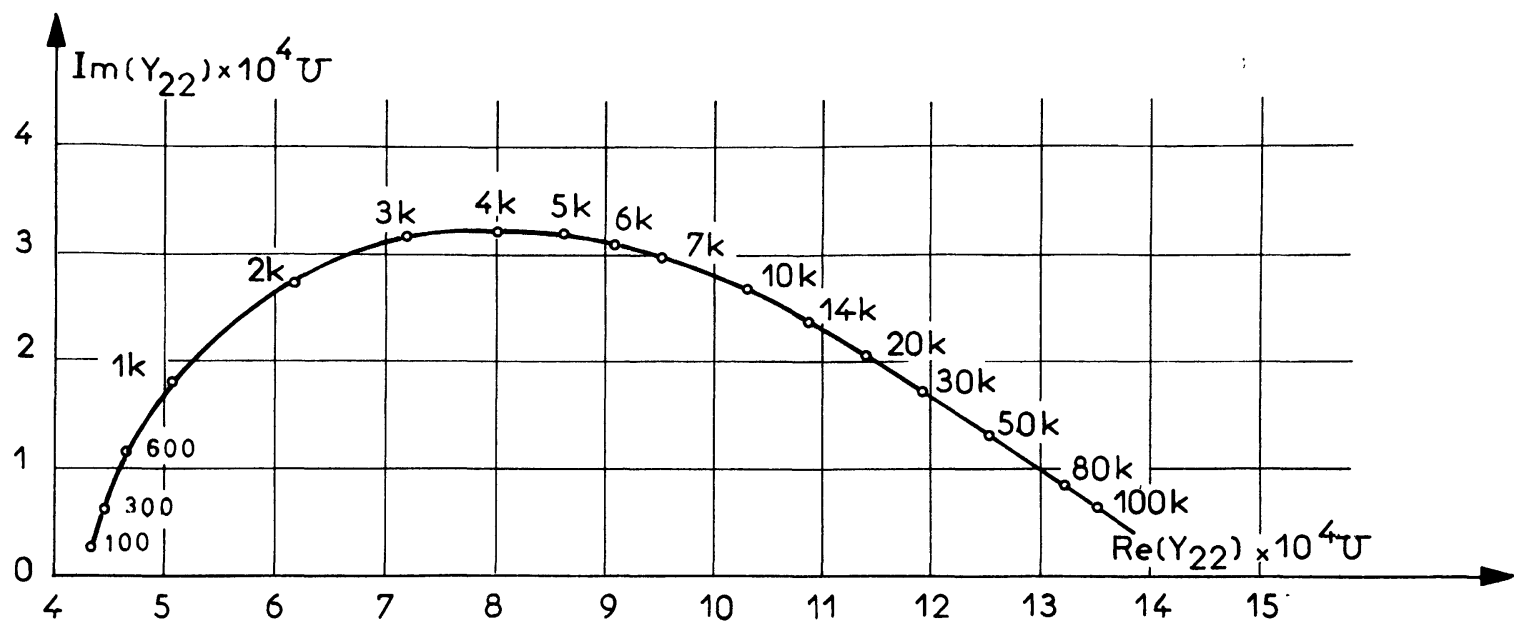

Fig. 4.4. - Variation de l'admittance de sortie en fonction de la fréquence. Structure avec grille GAT $4: V_{\mathrm{D}}=5 \mathrm{~V} ; V_{\mathrm{G}}=-1,8 \mathrm{~V}$; $I_{\mathrm{D}}=21 \mathrm{~mA} ; T_{\mathrm{a}}=300 \mathrm{~K}$.

[Low frequency variation of the output admittance. MESFET GAT $4: V_{\mathrm{D}}=5 \mathrm{~V} ; V_{\mathrm{G}}=-1.8 \mathrm{~V} ; I_{\mathrm{D}}=21 \mathrm{~mA} ; T_{\mathrm{a}}=300 \mathrm{~K}$.]
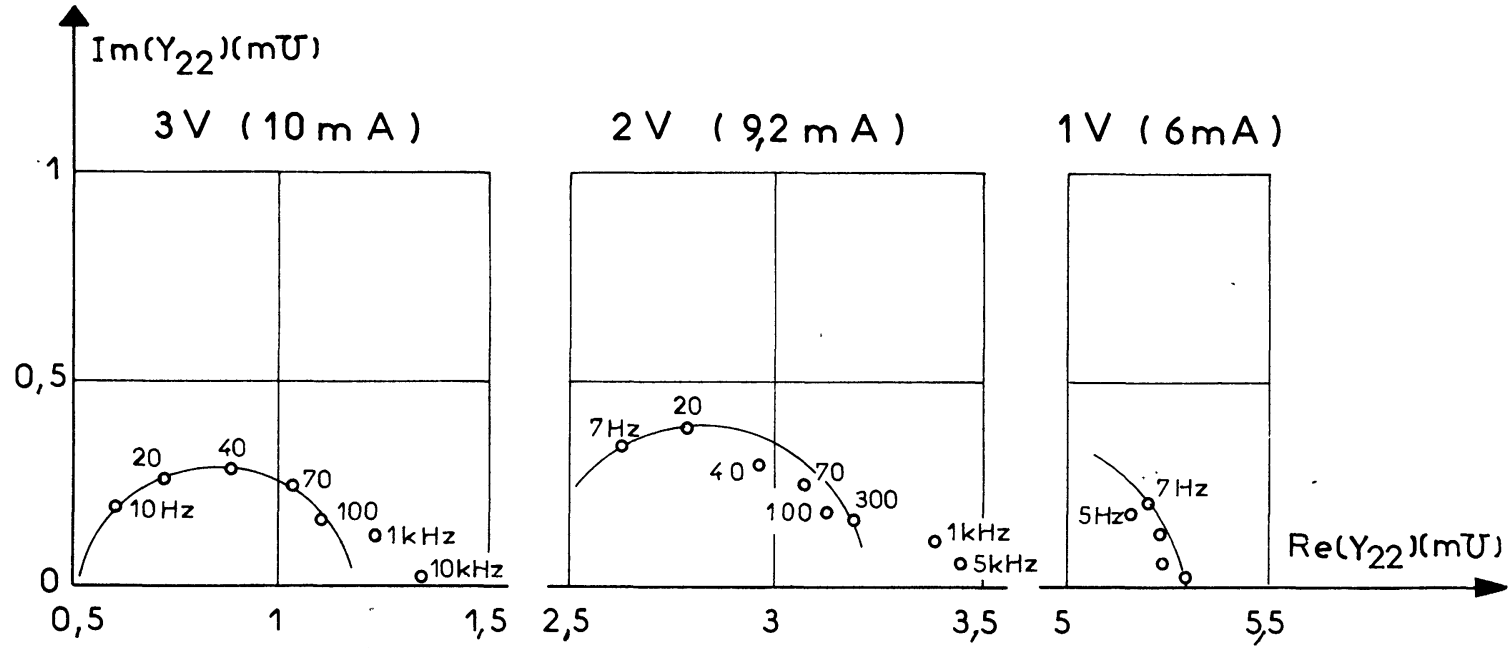

Fig. 5. - Variations de l'admittance de sortie en fonction de la fréquence. Structure sans grille TH no. $2 ; T_{\mathrm{a}}=300 \mathrm{~K}$.

[Low frequency variation of the output admittance. Ungated device TH no. $2 ; T_{\mathrm{a}}=300 \mathrm{~K}$.]

de la transadmittance directe par un piégeage des porteurs sur des centres volumiques dont la section de capture se modifie sous l'effet du champ électrique. Ce piégeage assisté par le champ ne se produirait que dans la zone pincée du canal ou ce champ peut être très élevé [10]. Cette hypothèse ne permet pas d'expliquer la relaxation observée expérimentalement en zone ohmique, à champ faible.

Ces diverses analyses ont été proposées en utilisant un modèle du transistor ne tenant pas compte de la présence du substrat semi-isolant. Des travaux récents, effectués par Barrera [2], par Lehovec et Zuleeg [4] et par Tanimoto et al. et Kaufmann et $a l$. [5] ont démontré théoriquement qu'il doit exister une double charge d'espace à l'interface semiconducteur-substrat. L'extension de celle-ci dans le semi- isolant et dans la zone épitaxiale varie avec les propriétés du substrat et est affectée par la répartition du potentiel établie par la tension drain-source. Récemment, N. Yokohama et al. [6] ont mis expérimentalement en évidence cette existence et les modifications de ces extensions par la polarisation de substrat. En régime dynamique, Barrera [2] a émis l'hypothèse que les régimes transitoires du courant sont dûs à la modification de la charge de la capacité de charge d'espace. Nous montrerons que ces hypothèses, l'existence de cette double charge et la cinétique de formation qui est fonction de la dépendance temporelle des tensions, permettent d'expliquer les phénomènes de relaxation.

3.2. ANALYSE PHÉNOMÉNOLOGIQUE. - L'origine physique de la formation de la double charge d'espace 


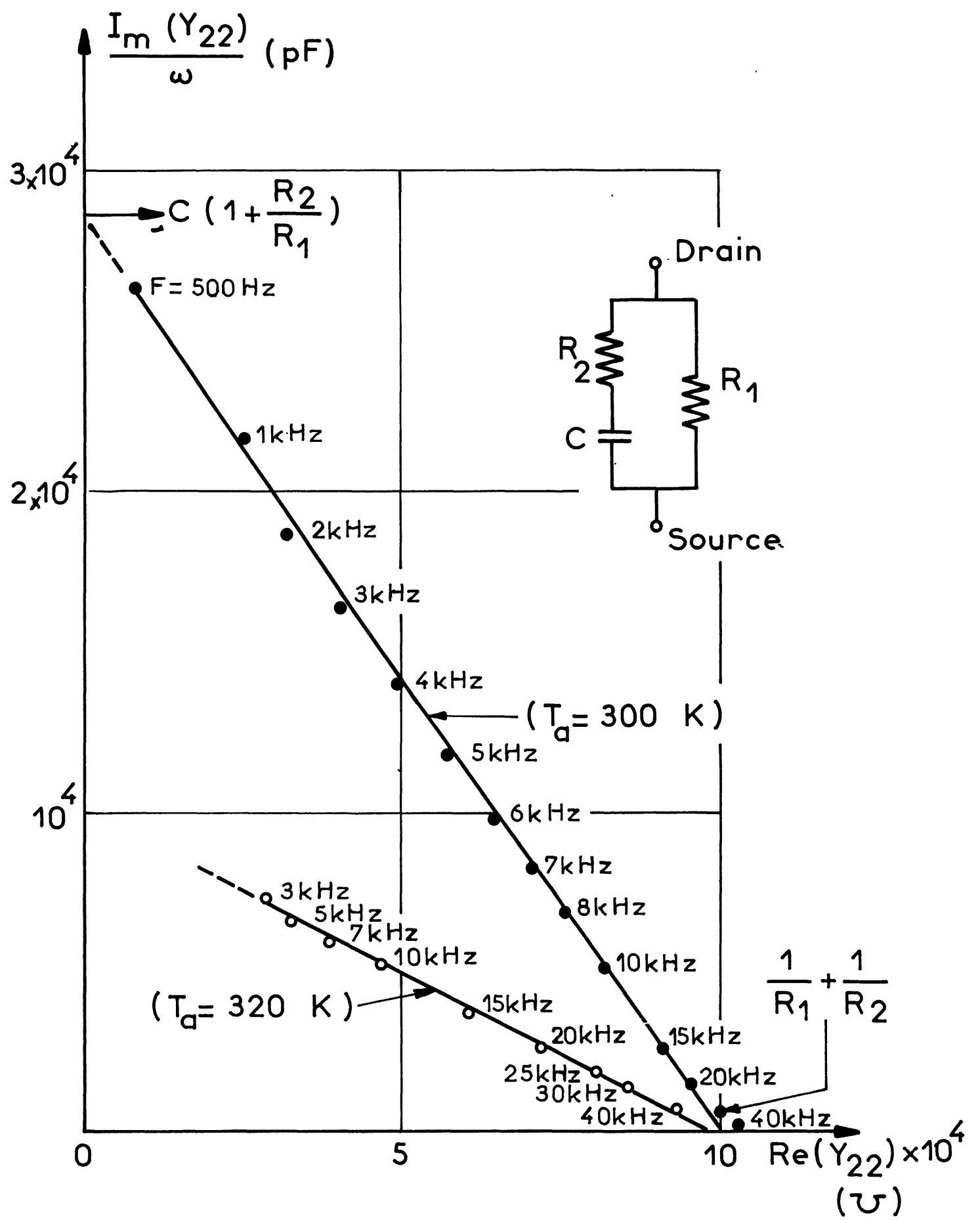

Fig. 6.1. - Méthode d'identification expérimentale de l'admittance de sortie à un réseau du premier ordre. Transistor avec grille GAT 2, 413 A $2 ; V_{\mathrm{D}}=5 \mathrm{~V} ; V_{\mathrm{G}}=0 \mathrm{~V}$.

[Experimental output admittance as identified to a first order network. MESFET GAT $2 ; V_{\mathrm{D}}=5 \mathrm{~V} ; V_{\mathrm{G}}=0 \mathrm{~V}$.]

à l'interface semiconducteur semi-isolant se déduit des conditions de polarisation dans les deux types de structures étudiées. Lorsque la tension de drain est positive par rapport aux deux électrodes réunies de source et de substrat, il s'établit une différence de potentiel positive entre tous point de la zone de conduction de coordonnée $y$ et le corps du substrat (Fig. 8). Cette différence de potentiel fait apparaître deux charges d'espace, positive dans le semiconduc- teur et négative dans le semi-isolant. La charge positive $Q_{1}(y)$ est due aux impuretés ionisées en densité $N_{\mathrm{D}}$ et s'étend sur une distance $x d(y)$ comptée à partir de l'interface. La charge d'espace négative $Q_{2}(y)$ est due à des centres qui peuvent s'ioniser dans le semiisolant par l'effet de la courbure des bandes d'énergie, due au potentiel électrostatique à l'interface. Ces centres sont ceux qui contribuent à rendre le substrat semi-isolant et dont le niveau d'énergie dans des 


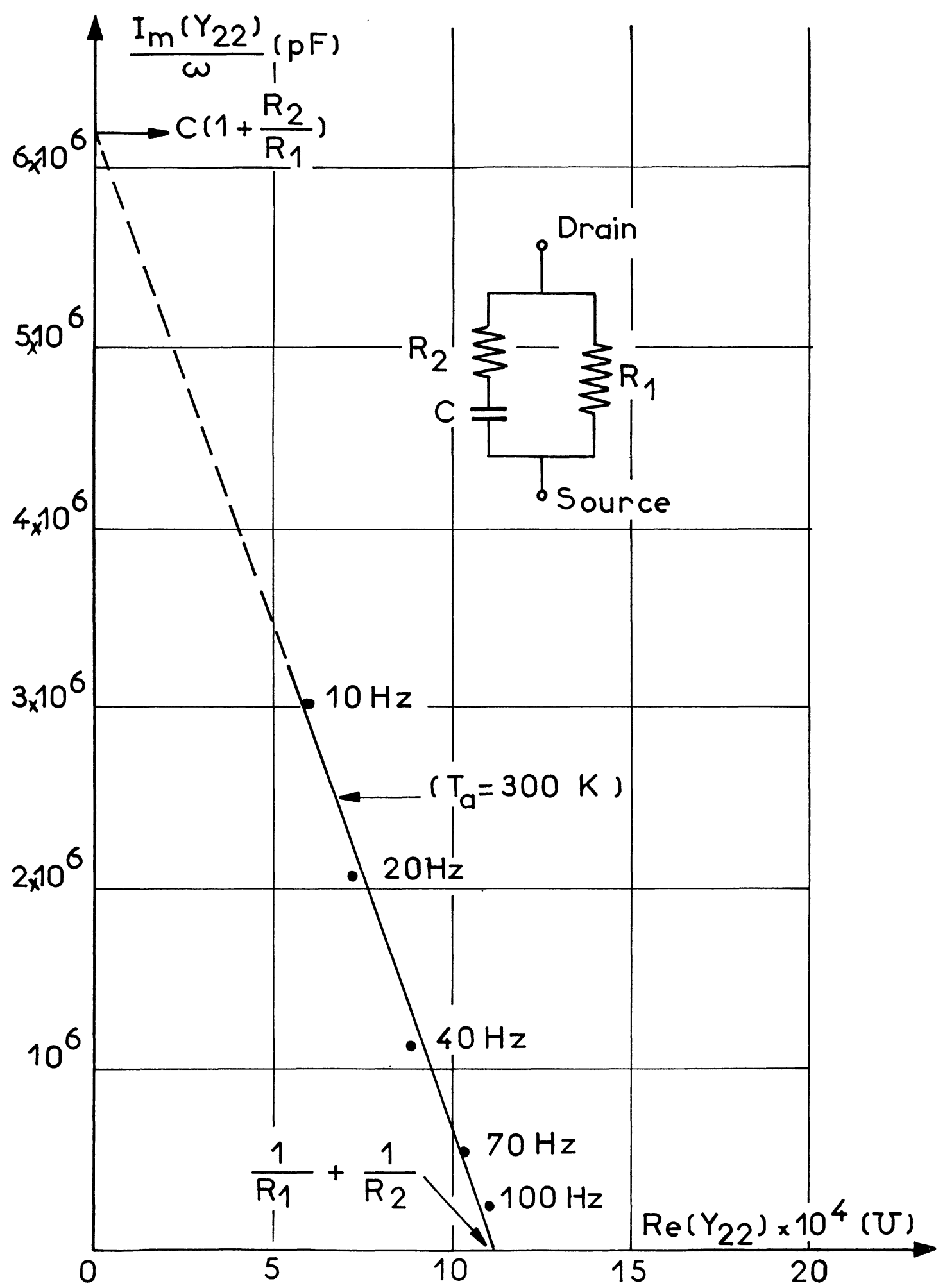

FIG. 6.2. - Méthode d'identification expérimentale de l'admittance de sortie à un réseau du $1^{\mathrm{er}}$ ordre. Structure sans grille $\mathbf{T H} ; V_{\mathbf{D}}=3 \mathrm{~V}$.

[Experimental output admittance as identified to a first order network. Ungated device TH; $V_{D}=3 \mathrm{~V}$.]

conditions de bandes plates est légèrement supérieur au niveau de Fermi. Leur densité sera notée $N_{\mathrm{t}}$. La distance sur laquelle s'étend la charge $Q_{2}(y)$ est appelée $x_{t}(y)$.

En régime d'équilibre, la charge positive $Q_{1}(y)$ est la charge image de $Q_{2}(y)$. La configuration élec- trostatique est identique à celle d'une jonction NP polarisée en inverse dont les dopants respectifs sont égaux à $N_{\mathrm{D}}$ et $N_{\mathrm{t}}$. En régime dynamique la neutralité reste respectée, mais la configuration des charges peut ne pas être en équilibre avec la répartition des tensions dans les structures. 


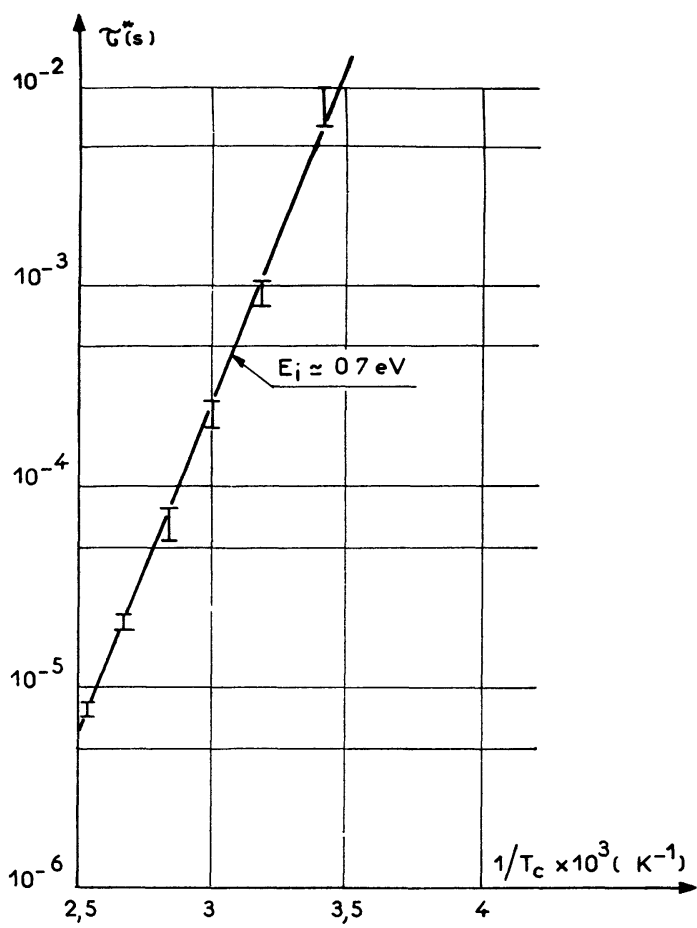

Fig. 7. - Variations de la constante de temps associée au diagramme circulaire en fonction de la température du cristal. Structure avec grille GAT $1 ; 318 \mathrm{~A}$.

[Variation of the time constant associated to the relaxation diagram as a function of the reciprocal chip temperature MESFET GAT 1.]

entre les deux couches. Par suite, l'épaisseur de la zone de conduction est réduite de cette épaisseur $x d(y)$. En faisant les hypothèses graduelles classiques
Dans les paragraphes suivants, les expressions des caractéristiques limites, courant drain-tension drain, seront explicitées et une analyse visant à décrire le diagramme circulaire de relaxation sera proposée. Il sera supposé intrinsèquement que les conditions de polarisation sont telles que les structures ne fonctionnent pas en régime de pincement, c'est-àdire que les conditions électrostatiques d'unidimensionnalité sont respectées au voisinage du drain $\left(\frac{\mathrm{d} E}{\mathrm{~d} y} \gg \frac{\mathrm{d} E}{\mathrm{~d} x}\right)$, et que les porteurs n'atteignent pas la vitesse de saturation.

3.2.1. Comportement en régime continu. - Pour des valeurs de tensions de polarisation continues fixées, les conditions de neutralité et d'équilibre à l'interface couche épitaxiale semi-isolant sont respectées. L'épaisseur de la zone dépeuplée dans la couche épitaxiale, obtenue par l'analogie avec la jonction NP, a pour expression [9] :

$$
x d(y)=\left[\frac{2 \varepsilon_{\mathrm{s}} \varepsilon_{\mathrm{o}}}{q N_{\mathrm{D}}}\left[V(y)+\Phi_{\mathrm{D}}\right] \frac{N_{\mathrm{t}}}{N_{\mathrm{t}}+N_{\mathrm{D}}}\right]^{1 / 2}
$$

$V(y)$ est le potentiel entre tout point du canal de conduction et la source, $\varepsilon_{\mathrm{s}}$ le facteur de permittivité $\mathrm{du}$ semiconducteur et. $\Phi_{\mathrm{D}}$ la tension de diffusion

et en négligeant les résistances d'accès aux régions actives, les courants satisfont aux équations différentielles :

$$
\begin{gathered}
I_{\mathrm{D}}=q N_{\mathrm{D}} Z d \mu(E y)\left[1-\frac{1}{V_{\mathrm{o}}^{1 / 2}}\left(\sqrt{V(y)+V_{\mathrm{bi}}-V_{\mathrm{G}}}-\alpha \sqrt{V(y)+\Phi_{\mathrm{D}}}\right)\right] \frac{\mathrm{d} V(y)}{\mathrm{d} y} \\
I_{\mathrm{D}}^{\prime}=q N_{\mathrm{D}} Z d \mu(E y)\left[1-\frac{\alpha}{V_{\mathrm{o}}^{1 / 2}} \sqrt{V(y)+\Phi_{\mathrm{D}}}\right] \frac{\mathrm{d} V(y)}{\mathrm{d} y}
\end{gathered}
$$

Ces relations s'appliquent respectivement au cas des structures avec et sans grille.

On a posé :

$$
\begin{gathered}
V_{\mathrm{o}}=\frac{q N_{\mathrm{D}} d^{2}}{2 \varepsilon_{\mathrm{o}} \varepsilon_{\mathrm{as}}} \\
\alpha=\left[\begin{array}{c}
N_{\mathrm{t}} \\
N_{\mathrm{t}}+N_{\mathrm{D}}
\end{array}\right]^{1 / 2}
\end{gathered}
$$

La loi $\mu(E y)$ représente la variation de mobilité des porteurs en fonction du champ électrique, $d$ est l'épaisseur de la couche épitaxiale, $V_{\mathrm{bi}}$ la hauteur de barrière métal de grille-semiconducteur, $V_{\mathrm{G}}$ la tension grille source et $q$ la charge électronique. Les expressions (2) et (3) diffèrent des relations classiques par l'introduction du terme $\alpha \sqrt{V(y)+\Phi_{\mathrm{D}}}$.
3.2.2. Existence des diverses caractéristiques limites. - L'analyse de la caractéristique de courant en larges signaux doit faire intervenir les formes et les dépendances temporelles de tensions sur les électrodes, ainsi que les lois qui régissent la cinétique de formation et de réponse de la charge $Q_{2}(y)$. Le but de ce paragraphe est de déterminer, dans le plan $I_{\mathrm{D}}\left(V_{\mathrm{D}}\right)$, les limites entre lesquelles se situent toutes les caractéristiques larges signaux. Le cas étudié qui est celui de la structure sans grille, où l'épaisseur de la zone de conduction est égale à $d-x_{\mathrm{d}}(y)$, est aisément transposable à celui du canal du transistor en considérant une nouvelle épaisseur $d-x_{\mathrm{d}}(y)-W(y)$, $W(y)$ étant la profondeur de la région dépeuplée sous la grille. Comme dans le paragraphe précédent seul le fonctionnement en régime graduel est analysé. 


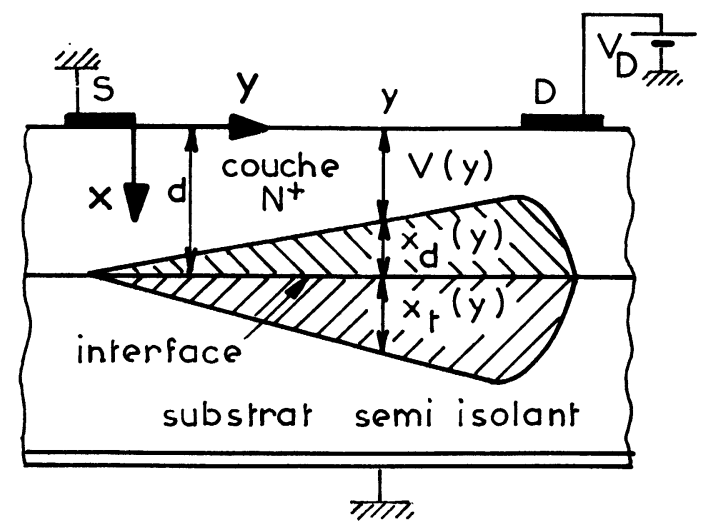

Coupe longitudinale

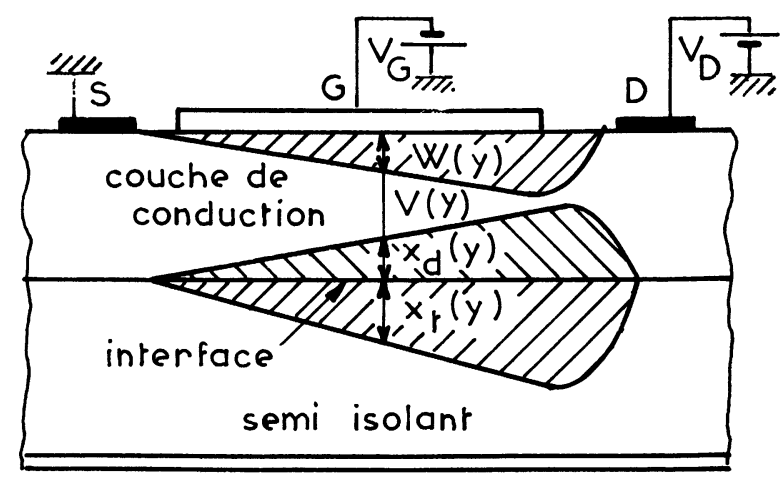

Coupe longitudinale

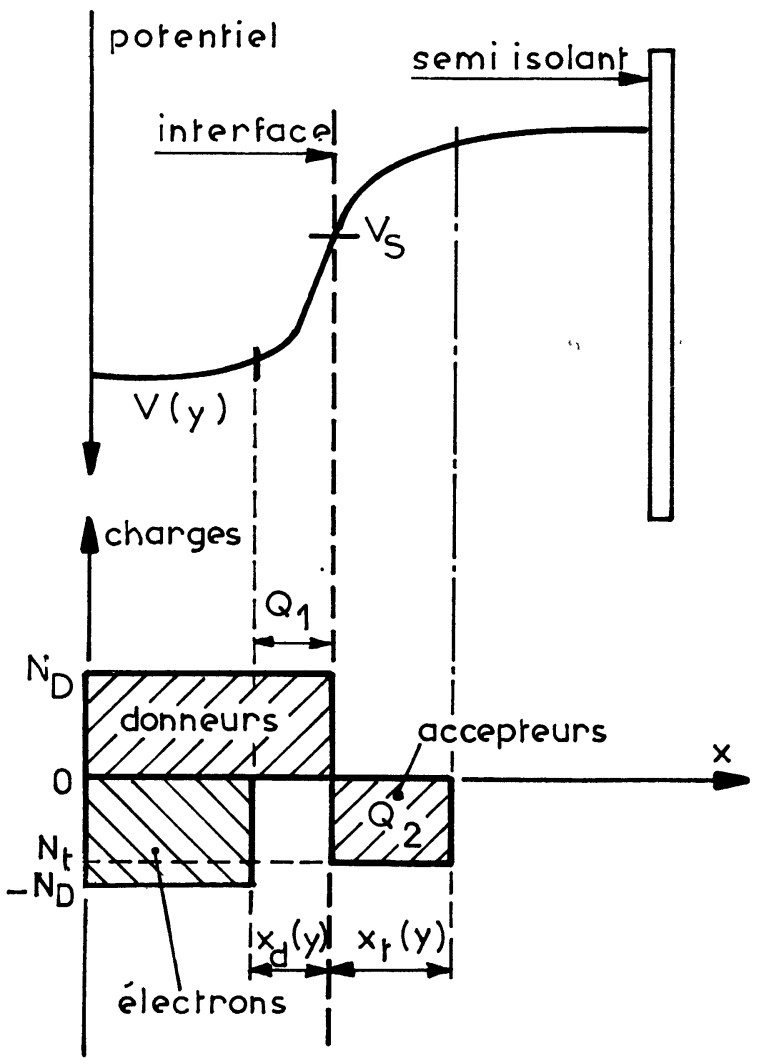

Coupe rransversale au point d'abscisse y

(a)

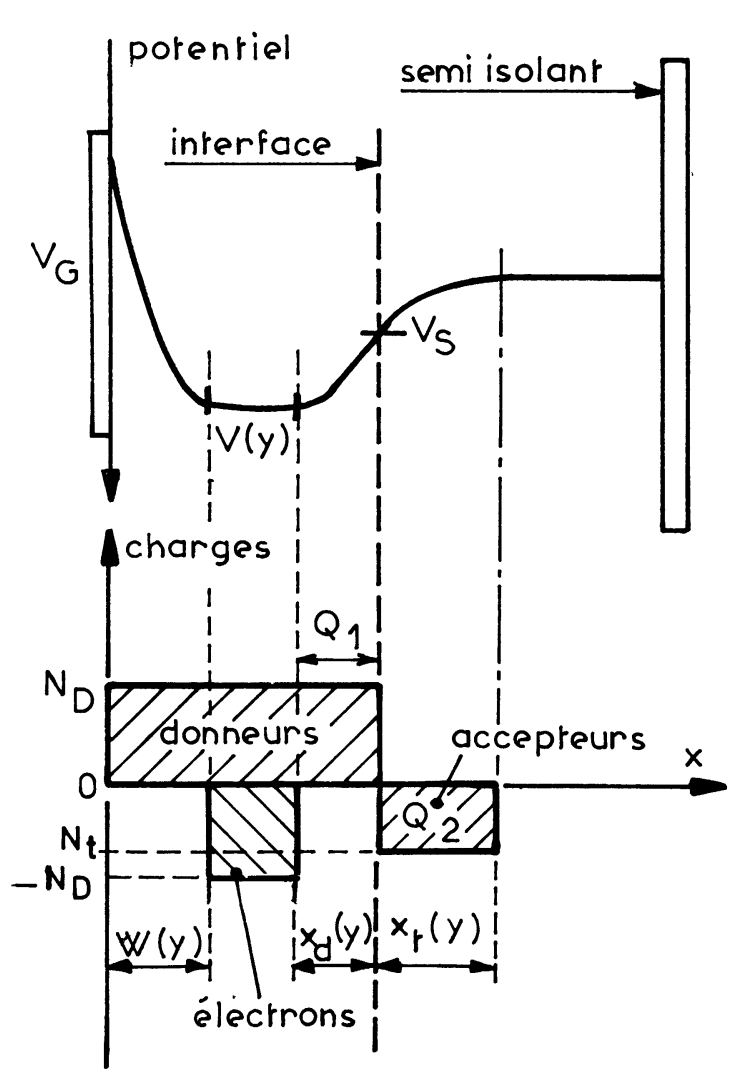

Coupe rransversale au point d'abscisse $y$.

(b)

Fig. 8. - Répartition des charges et du potentiel dans une structure sans grille (a) et avec grille (b).

[Depletion regions, potential, impurity distribution, and carrier distribution: a) in an ungated device, b) in a MESFET.] 
En régime dynamique, la charge image $Q_{1}(y)$ répond instantanément et est opposée à tout instant à $Q_{2}(y)$. La cinétique de formation de la charge $Q_{2}(y)$ dépend des probabilités de capture et d'émission de porteurs par les centres profonds. En régime impulsionnel de tension drain, cette charge $Q_{2}(y)$ ne peut suivre les variations du potentiel, elle est constante en fonction de la coordonnée $y$; l'épaisseur $x d$ reste constante égale à :

$$
x d=\sqrt{\frac{2 \varepsilon_{\mathrm{s}} \varepsilon_{\mathrm{o}}}{q N_{\mathrm{D}}} \cdot \bar{N}_{\mathrm{t}}+N_{\mathrm{D}}} \cdot \Phi_{\mathrm{D}}
$$

Pour une structure sans grille, le courant, en régime impulsionnel de tension drain entre les valeurs nulle et $V_{\mathrm{D}}$, est solution de l'équation différentielle :

$$
I_{\mathrm{D}_{0}}=q N_{\mathrm{D}} Z d \mu(E y) \cdot\left[1-\frac{\alpha}{V_{\mathrm{o}}^{1 / 2}} \Phi_{\mathrm{D}}^{1 / 2}\right] \frac{\mathrm{d} V}{\mathrm{~d} y}
$$

pour laquelle les conditions aux limites sont :

$$
\left\{\begin{array}{lll}
y=0 & \quad & V=0 \\
y=L & & V=V_{\mathbf{D}}
\end{array}\right.
$$

Pour une montée en tension drain entre les états 0 et $V_{\mathrm{D} 1}$, lente devant la durée d'extension de la charge $Q_{2}$, le courant pour la même structure est régi par l'équation différentielle :

$$
I_{\mathrm{D}_{1}}=q \cdot N_{\mathrm{D}} \cdot Z \cdot d \cdot \mu(E y) \cdot\left[1-\frac{\alpha}{V_{\mathrm{o}}^{1 / 2}} \cdot \sqrt{V_{1}+\Phi_{\mathrm{D}}}\right] \frac{\mathrm{d} V_{1}}{\mathrm{~d} y}
$$

dans laquelle les conditions aux limites sont :

$$
\begin{cases}y=0 & V_{1}=0 \\ y=L & V_{1}=V_{D}\end{cases}
$$

Pour une descente suffisamment rapide entre les états $V_{\mathrm{D}}$ et 0 , telle que la charge $Q_{2}(y)$ n'évolue pas, la caractéristique est régie par :

$$
I_{\mathrm{D}_{2}}=q \cdot N_{\mathrm{D}} \cdot Z \cdot d \cdot \mu(E y) \cdot\left[1-\frac{\alpha}{V_{\mathrm{o}}^{1 / 2}} \cdot \sqrt{ } V_{1}(y)+\Phi_{\mathrm{D}}\right] \cdot \frac{\mathrm{d} V_{2}}{\mathrm{~d} y}
$$

dans laquelle les conditions aux limites sont :

$$
\begin{cases}y=0 & V_{2}=0 \\ y=L & V_{2}=V_{\mathrm{D}}\end{cases}
$$

Dans cette caractéristique de retour, le potentiel $V_{1}(y)$ est celui qui est solution de l'équation différentielle (9) dans laquelle le courant $I_{\mathrm{D}_{1}}$ est pris pour la valeur de tension drain égale à $V_{\mathrm{D}_{1}}$.
Il n'est pas possible d'établir d'expression générale et analytique des solutions des équations différentielles précédentes puisqu'il est nécessaire de faire intervenir la loi de dépendance locale entre mobilité et champ électrique dans ces couches minces d'arséniure de gallium. Dans le cas où le champ Ey est suffisamment faible pour pouvoir admettre que la mobilité est constante, les diverses solutions s'écrivent :

$$
\begin{gathered}
I_{\mathrm{D}_{0}}=G_{\mathrm{o}}\left[1-\frac{\alpha \Phi_{\mathrm{D}}^{\mathrm{1} / 2}}{V_{\mathrm{o}}^{\mathrm{i} / 2}}\right] V_{\mathrm{D}} \\
I_{\mathrm{D}_{1}}=G_{\mathrm{o}}, V_{\mathrm{D}}-\frac{2}{3} \frac{1}{V_{\mathrm{o}}^{1 / 2}}\left[\alpha\left(V_{\mathrm{D}}+\Phi_{\mathrm{D}}\right)^{3 / 2}-\alpha \Phi_{\mathrm{D}}^{3 / 2}\right] \\
I_{\mathrm{D}_{2}}=G_{\mathrm{o}} \frac{V_{\mathrm{D}}}{V_{\mathrm{D}_{1}}}\left[V_{\mathrm{D}_{1}}-\frac{2}{3} \frac{\alpha}{V_{\mathrm{o}}^{1 / 2}}\left[\left(V_{\mathrm{D}_{1}}+\Phi_{\mathrm{D}}\right)^{3 / 2}-\Phi_{\mathrm{D}}^{3 / 2}\right]\right]
\end{gathered}
$$

où l'on a posé :

$$
G_{\mathrm{o}}=q \mu N_{\mathrm{D}} Z d / L
$$

Les allures des caractéristiques limites décrites par les relations (7) et (11) sont résumées sur la figure 9. Il existera par suite une infinité de caractéristiques possibles situées entre celles-ci et qui seront fonction à la fois de la dépendance temporelle de la tension drain. de la valeur maximale de celle-ci, et de la durée de stockage de l'échantillon dans les états initiaux et finaux.

3.2.3. Diagramme circulaire de relaxation. L'objectif de ce paragraphe est de faire apparaître que la variation dynamique locale de la charge $Q_{2}(y)$, associée à l'équation de transport écrite sous forme différentielle dans une tranche de canal, conduit, 


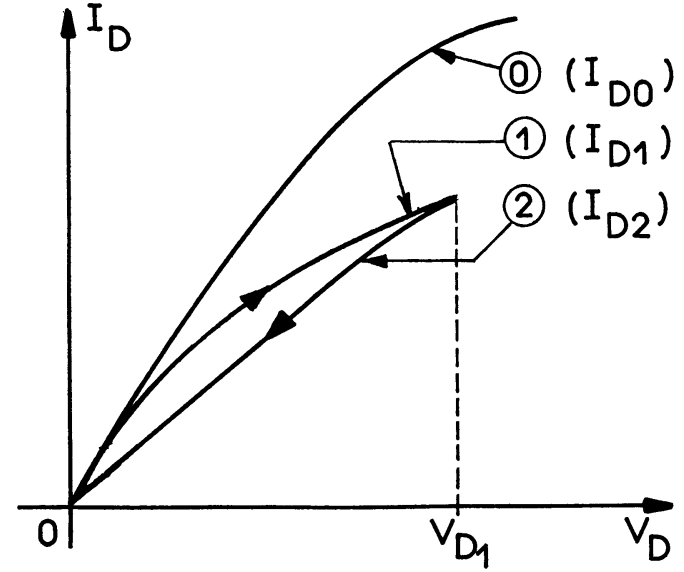

FIG. 9. - Allure des différentes caractéristiques limites. Structure sans grille (échelles arbitraires).

$0=$ régime impulsionnel (rel. (7)),

$1=$ durée de montée en $V_{\mathrm{D}}$ lente devant la durée de formation de la charge $Q_{2}: Q_{2}$ dépend de $V_{\mathrm{D}}$ (rel. (9)),

$2=$ durée de descente en $V_{\mathrm{D}}$ rapide devant la durée d'extinction de la charge $Q_{2}: Q_{2}$ n'est fonction que de $V_{\mathrm{D} 1}$ (rel. (11)).

[Current Voltage characteristics of an ungated device (arbitrary scales) : 0 . using pulse techniques (Eq. (7)), 1. the rise time of $V_{\mathrm{D}}$ is small in comparison with the built-up time of the charge $Q_{2}$; $Q_{2}$ depends on the voltage $V_{\mathrm{D}}$ (Eq. (9)), 2. $Q_{2}$ cannot follow the fall time of $V_{\mathrm{D}} ; Q_{2}$ is only dependent on the voltage $V_{\mathrm{D}_{1}}$ (Eq. (11)).]

après intégration de cette équation, à exprimer l'admittance de sortie sous la forme d'un réseau du premier ordre. Dans un but de simplification d'exposé l'analyse ne sera développée que pour les structures sans grille, dans le cadre d'hypothèse graduelle et de mobilité constante.
La modification fréquentielle de la conductance de sortie autour d'un point continu $\left(V_{\mathrm{D}}, I_{\mathrm{D}}\right)$ de polarisation est due à la réponse dynamique de la charge $Q_{2}(y, \omega)$ sous l'effet de la modulation du potentiel de canal imposée par les conditions d'excitation au drain. Afin de rendre compte localement des variations $\tilde{x} d(y, \omega)$, de la largeur du canal, en tout point d'abscisse $y$, sous l'effet de la modulation alternative sinusoïdale à la pulsation $\omega$ du potentiel, nous admettrons que la variation de la charge dans le substrat est régie par une loi à une seule constante de temps $\mathcal{C}$. On désignera par $\tilde{q}_{2}(\omega)$ cette variation sinusoïdale de charge autour de la valeur moyenne $Q_{2}\left[V_{\mathrm{s}}(y)\right]$ et par $\tilde{v}_{\mathrm{s}}$ les variations sinusoïdales du potentiel d'interface substrat couche épitaxiale autour de la valeur moyenne $V_{\mathrm{s}}(y)$. Cette variation est écrite sous la forme :

$$
\tilde{q}_{2}(\omega)=\frac{\tilde{v}_{\mathrm{s}} \cdot C\left[V_{\mathrm{s}}(y)\right]}{1+j \omega \mathscr{C}}
$$

$C\left[V_{\mathrm{s}}(y)\right]$ est la capacité dynamique en basses fréquences entre l'interface et le corps du substrat. En désignant par $\Delta Q_{2} m$ l'incrément de la charge $Q_{2}$ dû à l'effet de l'application d'un incrément de polarisation stationnaire égal au module $\left|\tilde{v}_{\mathrm{s}}\right|$ de $\tilde{v}_{s}$, cette capacité dynamique s'exprime par :

$$
C\left[V_{\mathrm{s}}(y)\right]=\frac{\Delta Q_{2} m\left[V_{\mathrm{s}}(y),\left|v_{\mathrm{s}}\right|\right]}{\left|v_{\mathrm{s}}\right|}
$$

De l'égalité entre la variation $\tilde{q}_{2}(\omega)$ et sa charge image induite dans la zone épitaxiale, on déduit la variation fréquentielle de l'épaisseur $x d$ :

$$
\tilde{x} d(y, \omega)=\alpha \sqrt{\frac{\varepsilon_{\mathrm{s}} \varepsilon_{\mathrm{o}}}{2 q N_{\mathrm{D}} \cdot\left(V(y)+\Phi_{\mathrm{D}}\right)}} \frac{\tilde{v}_{\mathrm{s}}}{1+j \omega \ell \alpha^{2}}
$$

Par ailleurs, la continuité du flux du courant dans une tranche différentielle de canal de conduction se traduit par l'expression suivante de la modulation $\tilde{i}(y, \omega)$ du courant de canal :

$\tilde{i}(y, \omega)=q \mu N_{\mathrm{D}} Z d\left\{\frac{\mathrm{d} \tilde{v}(y, \omega)}{\mathrm{d} y}\left[1-\alpha \sqrt{\frac{V(y)+\Phi_{\mathrm{D}}}{V_{\mathrm{o}}}}\right]-\frac{\mathrm{d} V(y)}{\mathrm{d} y} \cdot \frac{\alpha \cdot \tilde{v}(y, \omega)}{2\left(1+j \omega \zeta \alpha^{2}\right) \sqrt{V_{\mathrm{o}}\left(V(y)+\Phi_{\mathrm{D}}\right)}}\right\}$

En négligeant le potentiel de diffusion $\Phi_{\mathrm{D}}$ devant la tension continue de drain $V_{\mathrm{D}}$, cette équation peut être intégrée. Compte tenu des conditions aux limites :

$$
\left\{\begin{array}{l}
\tilde{i}(L, \omega)=\tilde{i d} ; \quad \tilde{v}(L, \omega)=\tilde{v} d \\
\tilde{v}(0, \omega)=0
\end{array}\right.
$$

l'admittance de sortie, définie par le rapport $\tilde{i} d / \tilde{v} d$, s'exprime par :

$$
Y_{22}(\omega)=\frac{I_{\mathrm{D}} \cdot \alpha^{2}(1+a) \cdot(1+2 a)}{2 a\left(\sqrt{V_{\mathrm{o}}}-\alpha \sqrt{V_{\mathrm{D}}}\right) \cdot\left[-a \sqrt{V_{\mathrm{o}}}-\alpha \sqrt{V_{\mathrm{D}}} \cdot(1+a)+\left(\sqrt{V_{\mathrm{o}}}-\alpha \sqrt{V_{\mathrm{D}}}\right)^{-\frac{1}{a}-1} \cdot a \cdot V_{\mathrm{o}}^{1+\frac{1}{2 a}}\right]}
$$




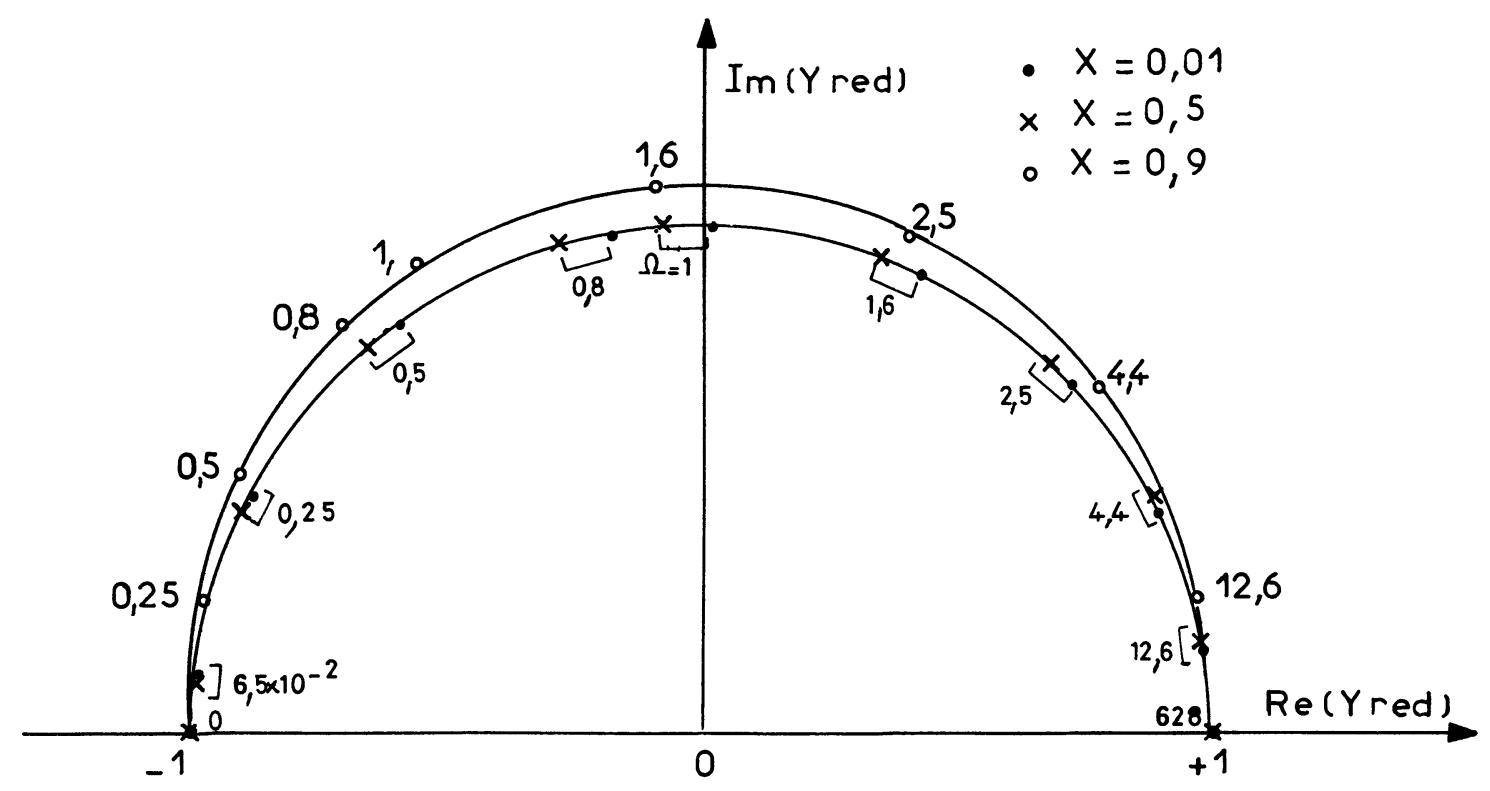

Fig. 10. - Variation de l'admittance normalisée en fonction de la pulsation réduite $\Omega$ et du paramètre $\chi$.

[ Normalized output admittance versus normalized angular frequency $\Omega$ at different values of the parameter $\chi$. ]

Le paramètre $a$ est défini par :

$$
a=1+j \omega \overparen{C} \alpha^{2}
$$

$$
X=\alpha \sqrt{\frac{V_{\mathrm{D}}}{V_{\mathrm{O}}}}
$$

Sur la figure 10, a été reporté le diagramme d'admittance normalisée, définie par :

et du paramètre de normalisation de fréquence :

$$
Y_{\mathrm{red}}=\frac{2 V_{\mathrm{D}}}{I_{\mathrm{D}}}\left[Y_{22}(\omega)-\frac{Y_{22 \mathrm{HF}}+Y_{22 \mathrm{BF}}}{2}\right]
$$

$$
\Omega=\omega \cdot \boldsymbol{G} \cdot \alpha^{2}
$$

$Y_{22 \mathrm{HF}}$ et $Y_{22 \mathrm{BF}}$ sont les valeurs limites de l'admittance $Y_{22}(\omega)$ aux fréquences infinie et nulle. Ce diagramme est un cercle tant que la variable $X$ ne dépasse pas la valeur 0,5 . Si cette condition est resen fonction de la variable réduite : pectée, la relation (22) peut être approximée par :

$$
Y_{22}(\omega)=-\frac{1}{3} \frac{\alpha \cdot I_{\mathrm{D}}}{2 \sqrt{V_{\mathrm{o}} V_{\mathrm{D}}}} \cdot \frac{1-j \omega \mathcal{C} \alpha^{2}}{1+j \omega \mathcal{C} \alpha^{2}}+\frac{Y_{22 \mathrm{HF}}+Y_{22 \mathrm{BF}}}{2}
$$

Le circuit équivalent de cette admittance est constitué par la mise en parallèle de deux branches. L'une est résistive; la valeur de sa résistance est égale à $\left(Y_{22 \mathrm{BF}}\right)^{-1}$. L'autre comprend une résistance $R_{2}$ et une capacité $C$ en série de valeurs respectives.

$$
\begin{gathered}
R_{2}=\frac{3 \cdot \sqrt{V_{\mathrm{O}} \cdot V_{\mathrm{D}}}}{\alpha \cdot I_{\mathrm{D}}} \\
C=\frac{\alpha^{3} \cdot \mathcal{C I} I_{\mathrm{D}}}{3 \sqrt{V_{\mathrm{D}} \cdot V_{\mathrm{O}}}}
\end{gathered}
$$

Le diamètre du diagramme circulaire est égal à :

$$
D=Y_{22 \mathrm{HF}}-Y_{2 \mathrm{BBF}}=\frac{1}{3} \frac{I_{\mathrm{D}}}{\sqrt{V_{\mathrm{O}} V_{\mathrm{D}}}} \sqrt{\frac{N_{\mathrm{t}}}{N_{\mathrm{t}}+N_{\mathrm{D}}}}
$$

et le maximum de la partie imaginaire se produit pour une valeur de la fréquence égale à :

$$
F_{\max }=\frac{N_{\mathrm{t}}+N_{\mathrm{D}}}{2 \pi \mathscr{C} N_{\mathrm{t}}}
$$

Dans le cadre de cette analyse, il apparaît que la constante de temps mesurée $\mathfrak{G}^{*}$ représente en fait la quantité :

$$
\mathfrak{C}^{*}=\mathfrak{C} \cdot \frac{N_{\mathrm{t}}}{N_{\mathrm{t}}+N_{\mathrm{D}}}
$$

et dépend donc du rapport des densités de dopant et de centres profonds. Cette relation fournit une première explication de la différence des valeurs des constantes de temps mesurées $\mathcal{C}^{*}$ sur les structures, 
du fait même que les dopages ne sont pas identiques. D'autre part, les constantes de temps variant exponentiellement avec la température de cristal, les valeurs mesurées de $\mathfrak{C}^{*}$ sont également fonction de la puissance continue dissipée par l'échantillon et de ses conditions de refroidissement.

Par suite, seule les connaissances précises de la température de cristal, des conditions de polarisation, du courant dans la structure, de ses caractéristiques technologiques - dopage et épaisseur - associées à la mesure expérimentale de la fréquence $F_{\max }$ et de la résistance $R_{2}$, par le diagramme circulaire, permettent de déterminer la constante de temps $\mathcal{C}$ et la densité $N_{\mathrm{t}}$ par les relations (28) et (29). Le tableau ci-dessous est un exemple de résultats obtenus sur trois couches différentes :

$\begin{array}{cccccc}\mathrm{N}^{\mathrm{o}} & & & & & \\ \text { couche } & N_{\mathrm{D}}\left(\mathrm{cm}^{-3}\right) & V_{\mathrm{D}}(\mathrm{V}) & I_{\mathrm{D}}(\mathrm{mA}) & V_{\mathrm{o}}(\mathrm{V}) & R_{2}(\Omega) \\ - & - & - & - & - & - \\ 1 & \sim 10^{16} & 2 & 8 & 0,8 & 1700 \\ 2 & \sim 10^{17} & 2 & 15 & 1,4 & 3100 \\ 3 & \sim 2 \times 10^{17} & 2 & 30 & 2,1 & 1400\end{array}$

Il apparaît que après correction due à la température, la constante de temps $\mathfrak{C}$ à l'ambiante est de l'ordre de quelques dixièmes de seconde, valeur notablement plus élevée que $\mathfrak{C}^{*}$, mesurée sur les diagrammes circulaires, qui évolue entre la microseconde et quelques millisecondes. Ces dernières valeurs sont, par ailleurs, à comparer aux constantes de temps mesurées, par d'autres auteurs, au moyen du régime transitoire de courant obtenu après application d'un échelon de tension sur le drain ou la grille [5] ou d'un échelon de tension négatif sur le substrat [6].

4. Conséquences et applications. - Le phénomène de relaxation que nous venons d'analyser peut avoir diverses conséquences et applications dont nous citerons celles qui nous paraissent essentielles. En premier lieu, à la température ambiante, la modification des caractéristiques courant-tension en fonction de la valeur maximale de la tension de drain et de la dépendance temporelle de celle-ci, peut avoir comme conséquence une évolution dans le temps des divers potentiels qui apparaissent dans les circuits logiques utilisant les structures planes, avec ou sans grille, à l'arséniure de gallium sur semi-isolant. Ces variations de niveaux se produiront avec des durées généralement longues devant les temps de commutation [11] des signaux logiques et devront être prises en compte dans la conception du circuit. Il sera nécessaire de déterminer dans quelle mesure ces instabilités lentes n'entraî-

$\begin{array}{rcccc}F_{\max }(\mathrm{Hz}) & T_{\mathrm{c}}(\mathrm{K}) & N_{\mathrm{t}}\left(\mathrm{cm}^{-3}\right) & \succ \text { à } T_{\mathrm{c}}(\mathrm{s}) & \begin{array}{c}\text { cà } 300 \mathrm{~K} \\ (\mathrm{~s})\end{array} \\ -29 & - & - & - & - \\ 500 & 340 & 8,7 \times 10^{14} & 0,078 & 0,5 \\ 100000 & 420 & 4,4 \times 10^{15} & 0,03 & 0,4 \\ & & & \end{array}$

neront pas une dégradation des valeurs des signaux logiques haut et bas.

Par ailleurs, une application immédiate du diagramme circulaire est la détermination des résistances thermiques des diverses structures [7]. Le principe est basé sur la mesure de l'évolution de la constante de temps associée à ce diagramme en fonction de la puissance dissipée par la structure. En désignant par $E_{\mathrm{c}}$ l'énergie d'activation thermique de cette constante de temps $\mathcal{G}^{*}$; $\operatorname{par} T_{\mathrm{c}}$ la température du cristal, par $P$ la puissance dissipée, par $T_{\mathrm{a}}$ la température ambiante et par $R_{\text {th }}$ la résistance thermique cristal-ambiante, on obtient:

$\mathfrak{G}^{*}=\mathfrak{G}_{\mathrm{o}} \exp \left(E_{\mathrm{c}} / k T_{\mathrm{c}}\right)$

avec: $T_{\mathrm{c}}=T_{\mathrm{a}}+R_{\mathrm{th}} . P$.

soit par développement limité:

$$
\log \mathfrak{C}^{*}=\log \mathscr{C}_{\mathrm{a}}^{*}-\frac{E_{\mathrm{c}}}{k T_{\mathrm{a}}} \frac{P}{T_{\mathrm{a}}} R_{\mathrm{th}}
$$

où $\mathfrak{C}_{\mathrm{a}}^{*}$ est la valeur de $\mathfrak{G}^{*}$ à la température ambiante, à puissance dissipée nulle. Le tracé des variations $d u$ logarithme de la constante de temps $\mathcal{G}^{*}$ en fonction de la puissance $P$ est linéaire (Fig. 11). De la pente de cette droite, on déduit la valeur de l'échauffement et de la résistance thermique $R_{\mathrm{th}}$. Le tableau ci-dessous indique les valeurs mesurées sur diverses structures:

$\begin{array}{ccc}\text { Type } & 7 \text { GF, } 6 & \text { GAT 1, 36.C.62 } \\ - & - & - \\ \text { Boîtier } & \text { Microstrip } & \text { TO } 72 \\ L \mu \mathrm{m} & 1 & 4 \\ R_{\text {th }} \mathrm{d}^{\circ} / \mathrm{W} & 860 & 360\end{array}$

5. Conclusion. - Dans cet article, les phénomènes basses fréquences qui apparaissent dans les couches épitaxiales et les transistors à effet de champ à l'arsé- niure de gallium réalisés sur substrat semi-isolant ont été étudiés expérimentalement au moyen des caractéristiques courant drain-tension drain, et de la conduc- 


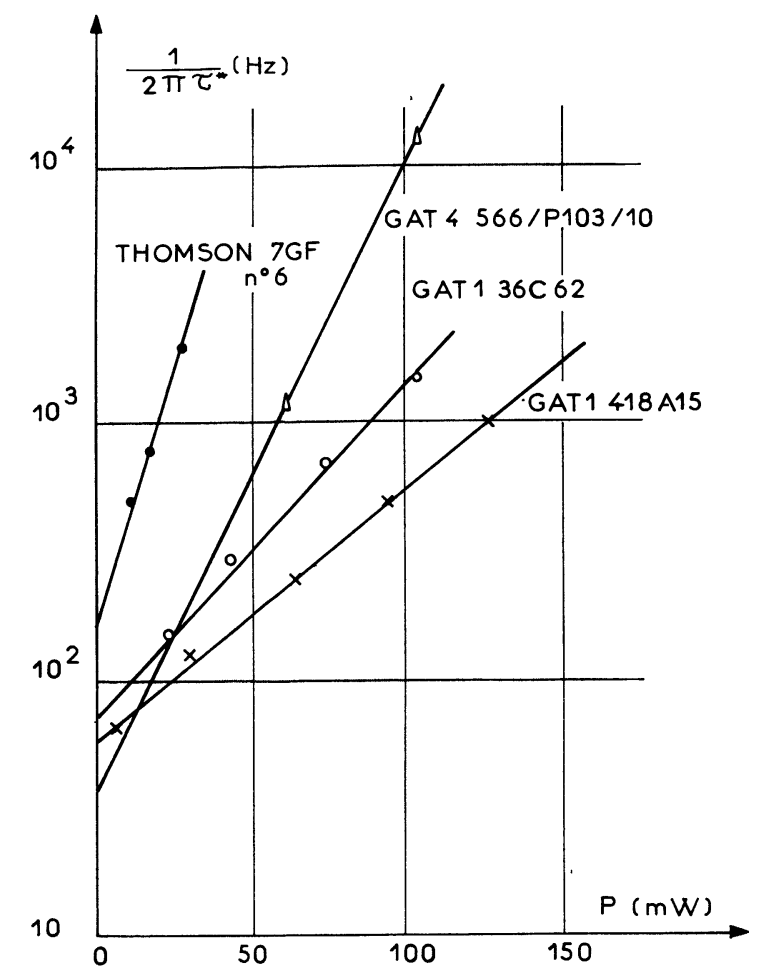

Fig. 11. - Variation des constantes de temps expérimentales en fonction de la puissance continue dissipée.

[Experimental time constant versus D.C. power.]

tance dynamique de sortie. Il a été montré que l'origine de ces mécanismes est liée à la formation d'une double charge d'espace à l'interface semiconducteur semi-isolant, dont les propriétés dépendent du potentiel de canal et des densités respectives de dopant et de centres profonds dans ces deux couches. En régime statique, les conditions de neutralité à cet interface, associées à l'équation de transport, impliquent une diminution du courant de drain par rapport au courant que l'on pourrait déduire des théories classiques. En régime dynamique, larges signaux, il est apparu que, suivant la nature et la forme de la tension appliquée sur le drain, il existe une infinité de caractéristiques courant-tension situées entre deux courbes limites. Une analyse phénoménologique a été proposée pour expliquer l'existence du diagramme complexe circulaire de l'admittance de sortie en petits signaux. La modification de cette admittance, due à la réponse des centres profonds, peut être traduite par l'adjonction aux bornes du composant d'un réseau du premier ordre. Dans celui-ci, la résistance et la capacité sont fonctions des tensions de polarisation, du courant et du rapport des densités de dopant et de centres profonds. Cette dépendance permet d'expliquer les valeurs élevées de la capacité qui apparaît dans ce schéma équivalent, et que l'on peut mesurer expérimentalement.

D'un point de vue pratique, il semble que ces phénomènes puissent entraîner des instabilités temporelles des circuits de commutation utilisant les structures planes à l'arséniure de gallium. Enfin, la modification du diagramme de relaxation sous l'effet de l'échauffement interne du composant peut constituer un moyen d'accès à la résistance thermique cristalambiante.

Sur un plan plus prospectif, cette première analyse doit être poursuivie et complétée suivant divers axes. Le plus important est assurément de caractériser les centres responsables de la relaxation - nature, propriétés - et de trouver les moyens technologiques propres à les éliminer. La difficulté de ce problème est due au fait que ces centres se trouvent au voisinage de l'interface semiconducteur semi-isolant, côté semiisolant, dont les propriétés physiques sont mal connues.

Le deuxième axe d'étude, correspondant à l'utilisation des structures planes épitaxiales avec grille (transistor à effet de champ) ou sans grille (résistance, charge saturable) est de généraliser les travaux effectués dans cet article, d'une part pour préciser, en fonction des conditions d'utilisation, l'évolution temporelle du courant consécutive à l'application de signaux variables dans le temps sur le drain, la grille et le substrat, d'autre part pour évaluer l'effet de la deuxième charge d'espace sur le phénomène de saturation ou de pincement de canal du côté du drain. Ces divers points ne pourront être résolus en toute rigueur que par analyse bidimensionnelle dynamique, qui devra inclure les phénomènes électrostatiques et de conduction associés au semi-isolant et au semiconducteur, les cinétiques de réponse des centres profonds, et les mécanismes d'échauffement en régime transitoire.

Remerciements. - Les auteurs remercient Messieurs Gibeau et Laviron de la Société THOMSON C.S.F., Messieurs Turner et Abott de la Compagnie Plessey pour la réalisation et la fourniture d'échantillons d'études ainsi que leurs collègues du L.A.A.S. et particulièrement Monsieur Martinot pour leurs commentaires, discussions et critiques au cours de la préparation de cet article. 


\section{Bibliographie}

[1] Nozaki T., Ogawa M., Terao H., Watanabe H., Multi Layer Epitaxial Technology for the Schottky barrier Ga As FET Gallium Arsen. and Relat. Comp. Inst. Phys. Conf. Ser. No. 24 (1975) ch. 2, 46-54.

[2] BARrera J., The Importance of Substrate Properties on Ga As FET performance 5th Bienn Cornell Elec. Conf. Sec II. 3 (1975) 135-144.

[3] Asai S., Ishioka S., Kurono H., TaKahashi S., Kodera H., Effects of Deep Centers on Microwave Frequency Characteristics of Ga As Schottky Barrier Gate FET. Proc. of the 4th Conf. on S.S. Dev. Tokyo 1972. Supplement to the $J$. Japan Soc. Appl. Phys. 42 (1973) 71.

[4] LeHOVEC K., ZuLEEG R., Mobility and Carrier Distributions at the Interface between semiconducting and semi-Insulating Gallium arsenide. Gallium Arsen. and Relat. Comp. Inst. Phys. Conf. Ser. No. 24 (1975) ch. 5, 292-306.

[5] Tanimoto M., Suzuki K., Itoh T., Ikoma T., Yanai H. et Kaufmann L.M.F., Nievendick W., Heime K. Effect of Interface Traps on the Ga As Planar Devices dans: Abstr. Devic. Res. Conf. 21-25 June 1976 - Univ. of UTAH ou: IEEE Trans. E.D. - Nov. $1976-1258-1259$ ou: 6 th EUR. S.S. Dev. Res. Conf.: München 1976, 80-81.

[6] Yokohama N., Shibatomi A., OhKawa S., Fukuta M., IshiKawa H., Doc. personnelle Fujitsue Lab. Lim. et Electrical Properties of the Interface Between N Ga As Epitaxial Layer and Cr. Doped Substrate. Proc. Noth. American Conf. on Ga As and Related Compounds 1976 (à paraître courant 1977).

[7] Rossel P., Graffeuil J., Azizi C., Tranduc H., Low Frequency Relaxation phenomena in Ga As MESFET, 6th EUR. S.S. Dev. Res. Conf. München 1976, 82-83.

[8] Adlerstein M. G., Electrical Traps in Ga As Microwave FET'S Electr. Lett. 12 (1976) 297.

[9] Grove A. S., Physics and technology of semiconductor Devices (John Wiley and Sons,) 1967, ch. 6.

[10] Liechti C. A., Microwave Field Effect Transistor - 1976 IEEE Trans. M.T.T. 24 (1976) 279.

[11] Van Tuyl R. L., Liechti C.A., High Speed Integrated Logic with Ga As MESFET'S J. S.S.C. 9 (1974) 269. 